\title{
Descent Calculations for the Elliptic Curves of Conductor 11
}

\author{
Tom Fisher
}

April 18, 2001

\begin{abstract}
Let $A$ be one of the three elliptic curves over $\mathbf{Q}$ with conductor 11. We show that $A$ has Mordell-Weil rank zero over its field of 5division points. In each case we also compute the 5-primary part of the Tate-Shafarevich group. Our calculations make use of the Galois equivariance of the Cassels-Tate pairing.
\end{abstract}

\section{Introduction}

Ever since the work of Mazur [Ma] the elliptic curves of conductor 11 have provided a testing ground for the Iwasawa theory of elliptic curves. We recall from [V1] that these curves form a single isogeny class, and have explicit Weierstrass equations

$$
\begin{array}{lll}
A_{0}=X_{0}(11) & y^{2}+y=x^{3}-x^{2}-10 x-20 & 11 \mathrm{~A} 1 \\
A_{1}=X_{1}(11) & y^{2}+y=x^{3}-x^{2} & 11 \mathrm{~A} 3 \\
A_{2} & y^{2}+y=x^{3}-x^{2}-7820 x-263580 & 11 \mathrm{~A} 2
\end{array}
$$

Here the labels 11A1-3 are those used in [Cr], whereas the labels $A_{0}, A_{1}, A_{2}$ are taken from [CS]. When there is no need to distinguish the three curves we shall simply write $A$ to denote any one of them.

Coates and Howson $[\mathrm{CH}]$ have used the elliptic curves of conductor 11 to illustrate their work on non-abelian Iwasawa theory. A natural question to ask is

How does the Mordell-Weil rank behave as we pass up the tower of fields given by adjoining the 5-power division points? 
Although we are still unable to answer this question, we prove that the rank is zero over the field of 5-division points for each of the three curves.

It seems that Mazur [Ma, Cor. 9.10] was the first to show $\operatorname{rank} A(\mathbf{Q})=0$ and $\amalg(A / \mathbf{Q})(5)=0$. An extension of this result to $\mathbf{Q}\left(\mu_{5}\right)$, due to Greenberg, may be found in [CS]. In each case the authors put their classical descent calculations to good use in studying the behaviour of Selmer groups over the cyclotomic $\mathbf{Z}_{5}$-extension. For instance in $[\mathrm{CS}]$ it is shown that $\operatorname{rank} A\left(\mathbf{Q}\left(\mu_{5^{\infty}}\right)\right)=0$. It is hoped that our results will have equally striking applications.

The curves of conductor 11 are chosen since they appear first in the list of modular curves, and they do not admit complex multiplication. The prime 5 is chosen to make the problem more tractable. Indeed there are isogenies of degree 5 defined over $\mathbf{Q}$

$$
A_{1} \rightleftarrows A_{0} \rightleftarrows A_{2}
$$

The curves $A_{0}$ and $A_{1}$ each have a rational point of order 5 , whereas $A_{2}$ does not. By properties of the Weil pairing we deduce $A_{0}[5] \simeq \mu_{5} \oplus \mathbf{Z} / 5 \mathbf{Z}$ as a Galois module. Furthermore there are exact sequences

$$
0 \rightarrow \mathbf{Z} / 5 \mathbf{Z} \rightarrow A_{1} \rightarrow A_{0} \rightarrow 0 \quad 0 \rightarrow \mu_{5} \rightarrow A_{2} \rightarrow A_{0} \rightarrow 0
$$

The fields of 5-division points are $k=\mathbf{Q}\left(\mu_{5}\right), K_{1}=\mathbf{Q}\left(A_{1}[5]\right)$ and $K_{2}=$ $\mathrm{Q}\left(A_{2}[5]\right)$. Since $K_{1}$ and $K_{2}$ are non-abelian and of degree 20 , it should come as no surprise that our descent calculations are rather more involved than those cited above. Our conclusions are

Theorem 1 Let $K_{1}=\mathbf{Q}\left(A_{1}[5]\right)$. Then $\operatorname{rank} A\left(K_{1}\right)=0$ and

$$
\begin{array}{ll}
A_{0}\left(K_{1}\right) \simeq(\mathbf{Z} / 5 \mathbf{Z})^{2} & \amalg\left(A_{0} / K_{1}\right)(5) \simeq(\mathbf{Z} / 5 \mathbf{Z})^{8} \\
A_{1}\left(K_{1}\right) \simeq(\mathbf{Z} / 5 \mathbf{Z})^{2} & \\
A_{2}\left(K_{1}\right) \simeq \mathbf{Z} / 5 \mathbf{Z} & \amalg\left(A_{1} / K_{1}\right)(5) \simeq(\mathbf{Z} / 5 \mathbf{Z})^{2} \\
&
\end{array}
$$

Theorem 2 Let $K_{2}=\mathbf{Q}\left(A_{2}[5]\right)$. Then $\operatorname{rank} A\left(K_{2}\right)=0$ and

$$
\begin{array}{ll}
A_{0}\left(K_{2}\right) \simeq(\mathbf{Z} / 5 \mathbf{Z})^{2} & \amalg\left(A_{0} / K_{2}\right)(5) \simeq(\mathbf{Z} / 5 \mathbf{Z})^{8} \\
A_{1}\left(K_{2}\right) \simeq \mathbf{Z} / 5 \mathbf{Z} & \amalg\left(A_{1} / K_{2}\right)(5)=0 \\
A_{2}\left(K_{2}\right) \simeq(\mathbf{Z} / 5 \mathbf{Z})^{2} & \amalg\left(A_{2} / K_{2}\right)(5) \simeq(\mathbf{Z} / 5 \mathbf{Z})^{6} \oplus(\mathbf{Z} / 25 \mathbf{Z})^{8} .
\end{array}
$$


It is easy to check that these results are compatible with the isogeny invariance of the Birch Swinnerton-Dyer conjecture, as proved by Cassels [Ca3]. Let us note that for $\mathfrak{p} \mid 11$, inspection of the $j$-invariants shows that the Tamagawa factors are $c_{\mathfrak{p}}\left(A_{0}\right)=5 \operatorname{ord}_{\mathfrak{p}}(11)$ and $c_{\mathfrak{p}}\left(A_{1}\right)=c_{\mathfrak{p}}\left(A_{2}\right)=\operatorname{ord}_{\mathfrak{p}}(11)$. At each infinite place, it follows by Vélu's formulae [V2] that the periods $\Omega_{i}$ are related via $\Omega_{1} / \Omega_{0}=\Omega_{0} / \Omega_{2}=5$.

In $\S 1$ we introduce some subfields of $\mathbf{Q}\left(A\left[5^{\infty}\right]\right)$. In $\S 2$ we recall from [F0], [F1], a description of the Selmer groups attached to the 5-isogenies (1). The analogue of Theorems 1 and 2 for $k=\mathbf{Q}\left(\mu_{5}\right)$ is an easy consequence. In $\S 3$ we give explicit Kummer generators for the fields introduced in $\S 1$. In $\S 4$ we recall the definition of the Cassels-Tate pairing. Following the work of McCallum [Mc] and Beaver [B] we give a formula for the pairing in the case we need. In $\S 5$ we discuss certain Galois modules, and the alternating pairings they admit. Finally in $\S 6$ and $\S 7$ we give the descent calculations proving Theorems 1 and 2 .

We have made extensive use of the computer algebra package pari in the course of this work. However we have striven where possible to give arguments that may be checked by hand. For the proof of Theorem 1 this goal has largely been achieved. In contrast the proof of Theorem 2 relies on us exhibiting a "non-trivial" unit in $K_{1} K_{2}$. Our method here was to ask pari to find all units in a certain degree 25 subfield. (This took 1 hour and 20 minutes on a $800 \mathrm{MHz}$ Pentium-III with $128 \mathrm{Mb}$ RAM.)

In a separate note [F2] we prove an analogue of Theorems 1 and 2 for the field $J_{1}=\mathbf{Q}\left(\mu_{5}\right) \mathbf{Q}\left(\mu_{11}\right)^{+}$. Again the rank is zero. Curiously our argument in that case does not require any formula for the Cassels-Tate pairing.

\section{Acknowledgements}

I would like to thank John Coates, Ralph Greenberg, Karl Rubin and Ed Schaefer for a number of valuable suggestions.

\section{Notation and Conventions}

For $F$ a perfect field we write $G_{F}:=\operatorname{Gal}(\bar{F} / F)$ and $H^{i}(F,-)=H^{i}\left(G_{F},-\right)$. By Hilbert's theorem 90 we identify $H^{1}\left(F, \mu_{5}\right)=F^{*} / F^{* 5}$. The number field $F$ has ring of integers $\mathfrak{O}_{F}$, unit group $\mathfrak{O}_{F}^{*}$, and class group $\mathfrak{C l}_{F}$. The local field $F_{\mathfrak{p}}$ has ring of integers $\mathfrak{O}_{\mathfrak{p}}$ and normalised valuation $\operatorname{ord}_{\mathfrak{p}}$. Since our 
interest is in descent via isogenies of odd degree we ignore the infinite places throughout.

Let $C$ and $D$ be elliptic curves defined over $F$, and let $\psi: C \rightarrow D$ be an isogeny of degree $m$. The Kummer exact sequence restricts to

$$
0 \longrightarrow D(F) / \psi C(F) \stackrel{\delta}{\longrightarrow} S^{(\psi)}(C / F) \longrightarrow \amalg(C / F)[\psi] \longrightarrow 0 .
$$

We frequently avoid giving our isogeny a name by writing $S(C \rightarrow D / F)$ for $S^{(\psi)}(C / F)$. The Weil pairing is denoted $e_{\psi}: C[\psi] \times D[\widehat{\psi}] \rightarrow \mu_{m}$.

The following notation relating to the field $k=\mathbf{Q}\left(\mu_{5}\right)$ is used throughout. We fix $\zeta$ a primitive 5 th root of unity and write $\operatorname{Ind}_{\zeta}: \mu_{5} \rightarrow \mathbf{Q} / \mathbf{Z}$ for the map $\zeta \mapsto 1 / 5$. Then $k$ has fundamental unit $\phi=1+\zeta+\zeta^{-1}$. (Taking $\zeta=\exp (2 \pi i / 5)$ this is the golden ratio.) We write $\bar{\phi}=-1 / \phi=1-\phi$ for its conjugate. In $\S 3$ we use $\phi$ to define involutions $\eta$ and $\varepsilon$ on $\mathbf{P}_{k}^{1}$. The primes of $k$ above 5 and 11 are $\mathfrak{l}=(1-\zeta)$ and $\mathfrak{p}_{i}=\left(\pi_{i}\right)$ where $\pi_{i}=2+\zeta^{i}$. We write $\omega: G_{\mathbf{Q}} \rightarrow(\mathbf{Z} / 5 \mathbf{Z})^{*}$ for the cyclotomic character.

\section{A description of $\operatorname{Gal}\left(\mathbf{Q}\left(A\left[5^{\infty}\right]\right) / \mathbf{Q}\right)$}

Serre $[$ Se2, §5.5] proved

Proposition 1.1 $\operatorname{Gal}\left(\mathbf{Q}\left(A\left[p^{\infty}\right]\right) / \mathbf{Q}\right) \simeq \mathrm{GL}_{2}\left(\mathbf{Z}_{p}\right)$ for all primes $p \neq 5$.

In contrast for $p=5, \mathbf{Q}\left(A\left[p^{\infty}\right]\right) / \mathbf{Q}\left(\mu_{p}\right)$ is a pro- $p$ extension. A description of the Galois group in this case was given by Lang and Trotter [LT]. In this section we present an alternative proof of their result and go on to compute the torsion subgroups listed as part of Theorems 1 and 2 .

Let $\mathcal{C}_{1}$ and $\mathcal{C}_{2}$ be the kernels of the degree 25 isogenies $A_{1} \rightarrow A_{2}$ and $A_{2} \rightarrow A_{1}$. We shall be concerned with the fields $J_{1}=k\left(\mathcal{C}_{1}\right), J_{2}=k\left(\mathcal{C}_{2}\right)$, $K_{1}=\mathbf{Q}\left(A_{1}[5]\right)$ and $K_{2}=\mathbf{Q}\left(A_{2}[5]\right)$.

Lemma 1.2 The fields $J_{1}, J_{2}, K_{1}, K_{2}$ are degree 5 Kummer extensions of $k$.

Proof. All is clear, except perhaps that these extensions are non-trivial. In fact $\mathcal{C}_{1}$ is generated by the cusps on $A_{1}=X_{1}(11)$ and these are defined over $\mathbf{Q}\left(\mu_{11}\right)^{+}=\mathbf{Q}\left(\mu_{11}\right) \cap \mathbf{R}$. Thus $J_{1}=\mathbf{Q}\left(\mu_{5}\right) \mathbf{Q}\left(\mu_{11}\right)^{+}$and $\mathbf{Q}\left(\mu_{25}\right) \subset J_{1} J_{2}$. For $K_{1}$ and $K_{2}$ we must show that the exact sequences (2) do not split as $G_{k^{-}}$ modules. As explained in [CS, Chapter 4] an examination of the Tate periods shows that these exact sequences do not even split as $G_{\mathbf{Q}_{11}}$-modules. 
We pick a basis $P, Q$ for the Tate module $T_{5}\left(A_{0}\right)$, such that the projections of $P$ and $Q$ in $A_{0}[5]$ generate $\operatorname{ker}\left(A_{0} \rightarrow A_{2}\right) \simeq \mathbf{Z} / 5 \mathbf{Z}$ and $\operatorname{ker}\left(A_{0} \rightarrow A_{1}\right) \simeq \mu_{5}$ respectively. Then the Galois representation $\rho: G_{\mathbf{Q}} \rightarrow \mathrm{GL}_{2}\left(\mathbf{Z}_{5}\right)$ attached to $A_{0}$ satisfies

$$
\rho(\sigma) \equiv\left(\begin{array}{cc}
1 & 0 \\
0 & \omega(\sigma)
\end{array}\right) \quad(\bmod 5)
$$

In particular

$$
\rho\left(G_{k}\right) \subset\left\{M \in \mathrm{GL}_{2}\left(\mathbf{Z}_{5}\right) \mid M \equiv I(\bmod 5)\right\} .
$$

Lemma 1.3 For $\sigma \in G_{k}$ let $\rho(\sigma)=I+5\left(\begin{array}{ll}a & b \\ c & d\end{array}\right)$. There are isomorphisms

$$
\begin{array}{rlrl}
\operatorname{Gal}\left(J_{1} / k\right) & \simeq \mathbf{Z} / 5 \mathbf{Z} ; \quad \sigma \mapsto a & & \operatorname{Gal}\left(K_{1} / k\right) \simeq \mathbf{Z} / 5 \mathbf{Z} ; \quad \sigma \mapsto b \\
\operatorname{Gal}\left(K_{2} / k\right) \simeq \mathbf{Z} / 5 \mathbf{Z} ; \quad \sigma \mapsto c & & \operatorname{Gal}\left(J_{2} / k\right) \simeq \mathbf{Z} / 5 \mathbf{Z} ; \quad \sigma \mapsto d .
\end{array}
$$

Furthermore, the action of $\operatorname{Gal}(k / \mathbf{Q})$ on these Galois groups is described by $\psi=1, \omega^{-1}, \omega$ and 1 respectively.

Proof. We check the first of these isomorphisms, the other cases being similar. Let $P_{r}, Q_{r}$ be the projections of $P, Q$ in $A_{0}\left[5^{r}\right]$. The image of $P_{2}$ under the 5 -isogeny $A_{0} \rightarrow A_{1}$ is a generator for $\mathcal{C}_{1}$. Thus for $\sigma \in G_{k}$

$$
\begin{aligned}
\sigma \text { fixes } J_{1} \text { pointwise } & \Longleftrightarrow \sigma\left(P_{2}\right)-P_{2} \in \operatorname{ker}\left(A_{0} \rightarrow A_{1}\right) \\
& \Longleftrightarrow a P_{1}+c Q_{1} \in \operatorname{ker}\left(A_{0} \rightarrow A_{1}\right) \\
& \Longleftrightarrow a \equiv 0 \quad(\bmod 5) .
\end{aligned}
$$

It follows that the map $\sigma \mapsto a$ induces an isomorphism $\operatorname{Gal}\left(J_{1} / k\right) \simeq \mathbf{Z} / 5 \mathbf{Z}$. Finally $\operatorname{Gal}(k / \mathbf{Q})$ acts on $G_{k}$ by conjugation, and we compute

$$
\left(\begin{array}{ll}
1 & 0 \\
0 & \omega
\end{array}\right)\left(\begin{array}{ll}
a & b \\
c & d
\end{array}\right)\left(\begin{array}{ll}
1 & 0 \\
0 & \omega
\end{array}\right)^{-1}=\left(\begin{array}{cc}
a & b \omega^{-1} \\
c \omega & d
\end{array}\right) .
$$

Lemma 1.4 The fields $J_{1}, J_{2}, K_{1}, K_{2}$ are independent degree 5 Kummer extensions of $k$. 
Proof. Given the distinct actions of $\operatorname{Gal}(k / \mathbf{Q})$ it suffices to check that $J_{1}$ and $J_{2}$ are independent. But $J_{1} J_{2}=\mathbf{Q}\left(\mu_{25}\right) \mathbf{Q}\left(\mu_{11}\right)^{+}$so this is clear.

The next proposition was originally proved by Lang and Trotter [LT, Part I, Theorem 8.1]. I am grateful to John Coates for pointing out to me the simpler proof presented here.

Proposition 1.5 The extension $\mathbf{Q}\left(A\left[5^{\infty}\right]\right) / \mathbf{Q}$ has Galois group

$$
\rho\left(G_{\mathbf{Q}}\right)=\left\{\left(\begin{array}{ll}
a & b \\
c & d
\end{array}\right) \in \mathrm{GL}_{2}\left(\mathbf{Z}_{5}\right) \mid\left(\begin{array}{ll}
a & b \\
c & d
\end{array}\right) \equiv\left(\begin{array}{ll}
1 & 0 \\
0 & *
\end{array}\right) \quad(\bmod 5)\right\} .
$$

Proof. We prove by induction on $r$ that the image of $\rho\left(G_{k}\right)$ in $\mathrm{GL}_{2}\left(\mathbf{Z} / 5^{r} \mathbf{Z}\right)$ is the kernel of the map $\mathrm{GL}_{2}\left(\mathbf{Z} / 5^{r} \mathbf{Z}\right) \rightarrow \mathrm{GL}_{2}(\mathbf{Z} / 5 \mathbf{Z})$. The case $r=2$ follows from Lemmas 1.3 and 1.4. The induction step is well known, and may be found in [LT] or [Se1]. It makes use of the identity

$$
\left(I+5^{r-1} M\right)^{5} \equiv I+5^{r} M \quad\left(\bmod 5^{r+1}\right) .
$$

We deduce that equality holds in (3), and the proposition follows.

In $\S 3$ we find explicit Kummer generators for the extensions $K_{1} / k$ and $K_{2} / k$. From these we learn that the prime above 5 is split in $K_{1} / k$ and is ramified in $K_{2} / k$. We prove the easy part of Theorems 1 and 2 .

Corollary 1.6 The torsion subgroups for $A\left(K_{1}\right)$ and $A\left(K_{2}\right)$ are

$$
\begin{aligned}
& A_{0}\left(K_{1}\right)_{\mathrm{tors}} \simeq(\mathbf{Z} / 5 \mathbf{Z})^{2} \quad A_{0}\left(K_{2}\right)_{\mathrm{tors}} \simeq(\mathbf{Z} / 5 \mathbf{Z})^{2} \\
& A_{1}\left(K_{1}\right)_{\text {tors }} \simeq(\mathbf{Z} / 5 \mathbf{Z})^{2} \quad A_{1}\left(K_{2}\right)_{\text {tors }} \simeq \mathbf{Z} / 5 \mathbf{Z} \\
& A_{2}\left(K_{1}\right)_{\text {tors }} \simeq \mathbf{Z} / 5 \mathbf{Z} \quad A_{2}\left(K_{2}\right)_{\text {tors }} \simeq(\mathbf{Z} / 5 \mathbf{Z})^{2} \text {. }
\end{aligned}
$$

Proof. Since $A$ has good reduction at 5 and $\widetilde{A}\left(\mathbf{F}_{5}\right) \simeq \mathbf{Z} / 5 \mathbf{Z}$, it suffices to check that the 5-power torsion is as claimed. We make the observation that $A_{0}$ has no point of order 25 defined over $K_{1} K_{2}$. Indeed if $\sigma \in G_{\mathbf{Q}}$ satisfies $\rho(\sigma)=6 I$ then $\sigma$ fixes pointwise the fields $K_{1}$ and $K_{2}$, but does not fix any point of order 25 on $A_{0}$. Thus $A_{0}\left(K_{i}\right)_{\text {tors }} \simeq(\mathbf{Z} / 5 \mathbf{Z})^{2}$ for $i=1,2$. Again for $i=1,2$ the inverse image of $A_{0}[5]$ under the 5-isogeny $A_{i} \rightarrow A_{0}$ has field of definition $J_{i} K_{i}$. The remaining statements now follow from Lemma 1.4.

The Selmer groups used in our calculations are of the most concrete nature, namely those attached to isogenies. They therefore contain contributions from torsion in the Mordell-Weil group. For this reason we make 
frequent implicit use of Corollary 1.6. For future reference we give another result on torsion subgroups.

Lemma 1.7 Let $\left[F: \mathbf{Q}_{11}\right]<\infty$. Then \# $A_{1}(F)(5) \leq 5\left[F: \mathbf{Q}_{11}\right]$.

Proof. We know that $A_{1}$ had multiplicative reduction, with Tamagawa factor $e=\operatorname{ord}(11)$. The number of smooth points over the residue field is $11^{f}-1$, and the multiplication by 5 map on the formal group is an isomorphism. Hence

$$
\# A_{1}(F)(5) \leq 5 e f=5\left[F: \mathbf{Q}_{11}\right]
$$

\section{Explicit descent via 5-isogeny}

The Selmer groups attached to the 5 -isogenies (1) are defined as subgroups of $H^{1}\left(F, \mu_{5}\right)=F^{*} / F^{* 5}$ and $H^{1}(F, \mathbf{Z} / 5 \mathbf{Z})=\operatorname{Hom}\left(G_{F}, \mathbf{Z} / 5 \mathbf{Z}\right)$.

Proposition 2.1 Let $F$ be a number field. Then

$$
\begin{aligned}
& S\left(A_{0} \rightarrow A_{1} / F\right) \simeq\left\{\theta \in F^{*} / F^{* 5} \mid \begin{array}{c}
\operatorname{ord}_{\mathfrak{p}}(\theta) \equiv 0(\bmod 5) \text { for all } \mathfrak{p} \\
\text { and } F(\sqrt[5]{\theta}) / F \text { split at } \mathfrak{p} \mid 11
\end{array}\right\} \\
& S\left(A_{1} \rightarrow A_{0} / F\right) \simeq\left\{\chi \in \operatorname{Hom}\left(G_{F}, \mathbf{Z} / 5 \mathbf{Z}\right) \mid \chi \text { unramified at all } \mathfrak{p} \nmid 11\right\} \\
& S\left(A_{2} \rightarrow A_{0} / F\right) \simeq\left\{\theta \in F^{*} / F^{* 5} \mid \operatorname{ord}_{\mathfrak{p}}(\theta) \equiv 0(\bmod 5) \text { for all } \mathfrak{p} \nmid 11\right\} \\
& S\left(A_{0} \rightarrow A_{2} / F\right) \simeq\left\{\chi \in \operatorname{Hom}\left(G_{F}, \mathbf{Z} / 5 \mathbf{Z}\right) \mid \begin{array}{c}
\chi \text { unramified at all } \mathfrak{p} \\
\text { and } \chi \text { split at } \mathfrak{p} \mid 11
\end{array}\right\}
\end{aligned}
$$

(Here $\chi$ split at $\mathfrak{p}$ means $\mathfrak{p}$ splits in the fixed field of the kernel of $\chi$.)

Proof. More generally in [F1] we considered pairs of 5-isogenous elliptic curves $C_{\lambda}$ and $D_{\lambda}$ with $\operatorname{ker}\left(C_{\lambda} \rightarrow D_{\lambda}\right) \simeq \mu_{5}$ and $\operatorname{ker}\left(D_{\lambda} \rightarrow C_{\lambda}\right) \simeq \mathbf{Z} / 5 \mathbf{Z}$. Explicitly $D_{\lambda}$ has Weierstrass equation

$$
y^{2}+(1-\lambda) x y-\lambda y=x^{3}-\lambda x^{2}
$$

and $\mathbf{Z} / 5 \mathbf{Z} \hookrightarrow D_{\lambda}(F)$ is generated by $(x, y)=(0,0)$. We see that $A_{1}=D_{1}$ and $A_{0}=D_{11}$. For each prime $\mathfrak{p}$ there is an exact sequence

$$
C_{\lambda}\left(F_{\mathfrak{p}}\right) \longrightarrow D_{\lambda}\left(F_{\mathfrak{p}}\right) \stackrel{\delta_{\mathfrak{p}}}{\longrightarrow} F_{\mathfrak{p}}^{*} / F_{\mathfrak{p}}^{* 5}
$$


We recall [F1, Propositions 2.15 and 2.16] that $\delta_{\mathfrak{p}}$ has image

$$
\operatorname{im} \delta_{\mathfrak{p}}=\left\{\begin{array}{cl}
F_{\mathfrak{p}}^{*} / F_{\mathfrak{p}}^{* 5} & \text { if } \operatorname{ord}_{\mathfrak{p}}(\lambda) \neq 0 \\
\mathfrak{O}_{\mathfrak{p}}^{*} / \mathfrak{O}_{\mathfrak{p}}^{* 5} & \text { if } \operatorname{ord}_{\mathfrak{p}}(\lambda)=\operatorname{ord}_{\mathfrak{p}}\left(\lambda^{2}-11 \lambda-1\right)=0 \\
1 & \text { if } \operatorname{ord}_{\mathfrak{p}}\left(\lambda^{2}-11 \lambda-1\right)>0 \text { and } \mathfrak{p} \nmid 5 .
\end{array}\right.
$$

The descriptions of $S\left(A_{0} \rightarrow A_{1} / F\right)$ and $S\left(A_{2} \rightarrow A_{0} / F\right)$ now follow on taking $\lambda=1$, respectively $\lambda=11$. Tate local duality tells us that the images of the local connecting maps attached to an isogeny and its dual are exact annihilators with respect to the Tate pairing. The descriptions of $S\left(A_{1} \rightarrow\right.$ $\left.A_{0} / F\right)$ and $S\left(A_{0} \rightarrow A_{2} / F\right)$ follow.

Suppose $F$ is number field for which we have a working knowledge of the unit group and the class group. It is now a straightforward exercise in Kummer theory to compute the Selmer groups $S\left(A_{0} \rightarrow A_{1} / F\right)$ and $S\left(A_{2} \rightarrow\right.$ $\left.A_{0} / F\right)$. If $\mu_{5} \subset F$, then the Selmer groups attached to the dual isogenies may be treated similarly. However there is a better way.

Proposition 2.2 Let $F$ be a number field with $r_{1}$ (resp. $r_{2}$ ), real (resp. pairs complex conjugate) embeddings and $m$ primes above 11 . Then

$$
\begin{aligned}
& \frac{\# S\left(A_{0} \rightarrow A_{1} / F\right)}{\# S\left(A_{1} \rightarrow A_{0} / F\right)}=\# \mu_{5}(F) \times 5^{r_{1}+r_{2}-1} \times 5^{-m} \\
& \frac{\# S\left(A_{2} \rightarrow A_{0} / F\right)}{\# S\left(A_{0} \rightarrow A_{2} / F\right)}=\# \mu_{5}(F) \times 5^{r_{1}+r_{2}-1} \times 5^{m} .
\end{aligned}
$$

Proof. This is an application of Cassels' formula [Ca3, Theorem 1.1]. The ratios of Tamagawa numbers are given in the introduction.

Remark 2.3 In simple cases, for example if $F$ has class number 1, it is a tolerable exercise in class field theory to deduce Proposition 2.2 directly from Proposition 2.1. The beauty of Cassels' formula is that the class number of $F$ does not appear.

Example 2.4 We use Propositions 2.1 and 2.2 to compute rank $A(k)$. We recall that $k$ has class number 1 , and that $\mathfrak{o}_{k}^{*}$ is generated by $\pm \zeta, \phi$, where $\phi=1+\zeta+\zeta^{-1}$. Writing $\pi_{i}=2+\zeta^{i}$ we find

$$
\begin{aligned}
& S\left(A_{0} \rightarrow A_{1} / k\right)=0 \quad \text { since } \zeta, \phi \notin\left(\mathfrak{o}_{k} / \pi_{1}\right)^{* 5} \\
& S\left(A_{1} \rightarrow A_{0} / k\right) \simeq(\mathbf{Z} / 5 \mathbf{Z})^{2} \quad \text { i.e. } \operatorname{Hom}\left(\operatorname{Gal}\left(J_{1} K_{1} / k\right), \mathbf{Z} / 5 \mathbf{Z}\right) \\
& S\left(A_{2} \rightarrow A_{0} / k\right) \simeq(\mathbf{Z} / 5 \mathbf{Z})^{6} \quad \text { i.e. }\left\langle\zeta, \phi, \pi_{1}, \pi_{2}, \pi_{3}, \pi_{4}\right\rangle \subset k^{*} / k^{* 5} \\
& S\left(A_{0} \rightarrow A_{2} / k\right)=0 \quad \text { since } h_{k}=1 \text {. }
\end{aligned}
$$


We deduce $\operatorname{rank} A(k)=0$ and $\amalg\left(A_{i} / k\right)(5)=0$ for $i=0,1$. We further find $\amalg\left(A_{2} / k\right)(5) \simeq(\mathbf{Z} / 5 \mathbf{Z})^{4}$.

\section{Torsion contributions and Kummer generators}

Let $C_{\lambda}$ and $D_{\lambda}$ be as in the proof of Proposition 2.1. Then $\lambda$ is a co-ordinate on $X_{1}(5) \simeq \mathbf{P}^{1}$ and this modular curve has cusps at $\lambda=0, \infty, \phi^{5}, \overline{\phi^{5}}$. There is an involution $\eta$ on $X_{1}(5)$, permuting the cusps, such that $\mu_{5} \hookrightarrow C_{\lambda}$ is isomorphic to $\mathbf{Z} / 5 \mathbf{Z} \hookrightarrow D_{\eta(\lambda)}$ over $F\left(\mu_{5}\right)$. We take

$$
\eta: \lambda \mapsto\left(\phi^{5} \lambda+1\right) /\left(\lambda-\phi^{5}\right) .
$$

For $\lambda \in F$ not a cusp of $X_{1}(5)$ there is a Kummer exact sequence

$$
0 \longrightarrow \mu_{5}(F) \longrightarrow C_{\lambda}(F) \longrightarrow D_{\lambda}(F) \stackrel{\delta}{\longrightarrow} F^{*} / F^{* 5} .
$$

Lemma 3.1 The image of $\mathbf{Z} / 5 \mathbf{Z} \hookrightarrow D_{\lambda}(F)$ under the connecting map $\delta$ is generated by $\lambda$.

Proof. In terms of the Weierstrass equation (4), the multiples of $(0,0)$ are $\left(\lambda, \lambda^{2}\right),(\lambda, 0)$, and $(0, \lambda)$. We recall from $[\mathrm{F} 1]$ that if $P=(x, y) \neq(0,0)$ then $\delta(P)=x y+y-x^{2}$. The lemma follows.

For $\lambda \in F$ we deduce $F\left(C_{\lambda}[5]\right)=F\left(\mu_{5}, \sqrt[5]{\lambda}\right)$. In particular $\eta(1)$ and 11 are Kummer generators for $K_{1} / k$ and $K_{2} / k$. We also learn that $X(5) \simeq \mathbf{P}^{1}$ with forgetful map

$$
X(5) \rightarrow X_{1}(5) ; \quad \tau \mapsto \tau^{5} .
$$

The cusps of $X(5)$ are at $\tau=0, \infty, \zeta^{i} \phi, \zeta^{i} \bar{\phi}$. Under stereographic projection these points may be viewed as the vertices of an icosahedron. There is an action of $\mathrm{PSL}_{2}(\mathbf{Z} / 5 \mathbf{Z}) \simeq A_{5}$ on $X(5)$ permuting the cusps, generated by $\tau \mapsto \zeta \tau$ and

$$
\varepsilon: \tau \mapsto(\phi \tau+1) /(\tau-\phi)
$$

Lemma 3.2 Suppose $\mu_{5} \subset F$. Let $\tau \in F$ and put $\lambda=\eta\left(\tau^{5}\right)$. Then the image of $(\mathbf{Z} / 5 \mathbf{Z})^{2} \hookrightarrow D_{\lambda}(F)$ under $\delta$ is generated by

$$
\lambda=\prod_{i=0}^{4} \varepsilon\left(\zeta^{i} \tau\right) \quad \text { and } \quad \prod_{i=0}^{4} \varepsilon\left(\zeta^{i} \tau\right)^{i} .
$$


Proof. In terms of the Weierstrass equation (4), $(\mathbf{Z} / 5 \mathbf{Z})^{2} \hookrightarrow D_{\lambda}(F)$ is generated by $(0,0)$ and

$$
\left(-\lambda \frac{(\zeta \tau-\phi)\left(\zeta^{4} \tau-\phi\right)}{(\phi \tau+1)(\tau-\phi)},-\lambda^{2} \frac{(\zeta \tau-\phi)\left(\zeta^{2} \tau-\phi\right)\left(\zeta^{4} \tau-\phi\right)^{2}}{(\phi \tau+1)^{2}(\tau-\phi)(\phi \zeta \tau+1)}\right)
$$

We conclude as in the proof of Lemma 3.1.

Remark 3.3 One way to construct the point (6) is to observe that in the notation of [F0], [F1] the curve

$$
T=T\left[\lambda ; \varepsilon(\tau), \varepsilon(\zeta \tau), \varepsilon\left(\zeta^{2} \tau\right), \varepsilon\left(\zeta^{3} \tau\right), \varepsilon\left(\zeta^{4} \tau\right)\right] \subset \mathbf{P}^{4}
$$

has rational point

$$
\left(\tau-\phi: \zeta \tau-\phi: \zeta^{2} \tau-\phi: \zeta^{3} \tau-\phi: \zeta^{4} \tau-\phi\right) .
$$

There is a diagonal action of $\mu_{5}$ on $T$ with quotient $D_{\lambda}$. In [F0, Appendix C] we give explicit equations for the map $T \rightarrow D_{\lambda}$ and this allows us to construct (6) from (7).

Applying Lemma 3.2 with $\tau=1$ gives Kummer generators for $J_{1} / k$ and $K_{1} / k$. Applying Lemma 3.2 with $\tau=\varepsilon(1)=-\phi^{3}$ gives Kummer generators for $J_{2} / k$ and $K_{2} / k$. We re-write these Kummer generators in terms of $\zeta, \phi, \pi_{1}, \pi_{2}, \pi_{3}, \pi_{4}$ and so obtain an alternative proof of Lemma 1.4.

\begin{tabular}{cccccc}
$L$ & \multicolumn{1}{c}{ Kummer generator for $L / k$} & $\psi^{-1} \omega$ & $\mathfrak{f}(L / k)$ & $d_{L}$ \\
\hline$J_{1}$ & $\prod \varepsilon\left(\zeta^{i}\right)^{i}$ & $\zeta^{2} \pi_{1} \pi_{2}^{3} \pi_{3}^{2} \pi_{4}^{4}$ & $\omega$ & $\mathfrak{p}_{1} \mathfrak{p}_{2} \mathfrak{p}_{3} \mathfrak{p}_{4}$ & $5^{15} 11^{16}$ \\
$K_{1}$ & $\eta(1)$ & $\phi^{2} \pi_{1} \pi_{2}^{4} \pi_{3}^{4} \pi_{4}$ & $\omega^{2}$ & $\mathfrak{p}_{1} \mathfrak{p}_{2} \mathfrak{p}_{3} \mathfrak{p}_{4}$ & $5^{15} 11^{16}$ \\
$K_{2}$ & $\eta\left(-\phi^{15}\right)$ & $\pi_{1} \pi_{2} \pi_{3} \pi_{4}$ & 1 & $\mathfrak{l}^{2} \mathfrak{p}_{1} \mathfrak{p}_{2} \mathfrak{p}_{3} \mathfrak{p}_{4}$ & $5^{23} 11^{16}$ \\
$J_{2}$ & $\prod \varepsilon\left(-\zeta^{i} \phi^{3}\right)^{i}$ & $\pi_{1} \pi_{2}^{3} \pi_{3}^{2} \pi_{4}^{4}$ & $\omega$ & $\mathfrak{l}^{5} \mathfrak{p}_{1} \mathfrak{p}_{2} \mathfrak{p}_{3} \mathfrak{p}_{4}$ & $5^{35} 11^{16}$
\end{tabular}

We recall that if $L / k$ is the Kummer extension corresponding to $\Delta \subset k^{*} / k^{* 5}$, then $\operatorname{Gal}(L / k) \simeq \operatorname{Hom}\left(\Delta, \mu_{5}\right)$ as a $\operatorname{Gal}(k / \mathbf{Q})$-module. Thus in the notation of Lemma 1.3, $\Delta$ is described by $\psi^{-1} \omega$. This is born out in our table.

The final two columns of our table record the conductor $\mathfrak{f}=\mathfrak{f}(L / k)$ and the absolute discriminant $d_{L}$. They are related via $d_{L}=(\text { Norm } \mathfrak{f})^{4} d_{k}^{5}$. It is clear that the primes above 11 ramify in each extension $L / k$. We determine the factorisation of the prime above 5 . 
(i) The extension $J_{1} / k$ is a translate of $\mathbf{Q}\left(\mu_{11}\right)^{+} / \mathbf{Q}$, so $\mathfrak{l}$ is inert.

(ii) The extension $K_{1} / k$ has Kummer generator

$$
\eta(1)=\frac{1+\phi^{5}}{1-\phi^{5}}=\overline{\phi^{5}}\left(1+\frac{\phi^{5}-\overline{\phi^{5}}}{1+\overline{\phi^{5}}}\right) .
$$

Since $\left(\phi^{5}-\overline{\phi^{5}}\right)^{2}=5^{3}$ the binomial theorem shows that $\eta(1)$ is a 5 th power in $k_{\mathfrak{l}}$. Thus $\mathfrak{l}$ splits in $K_{1} / k$.

(iii) The extension $K_{2} / k$ has Kummer generator 11 . The minimal polynomial for $\sqrt[5]{11}-1$ is an Eisenstein polynomial. Thus 5 is totally ramified in $K_{2} / \mathbf{Q}$. A useful intermediate step in computing $d_{K_{2}}$ is to show that $\mathbf{Q}(\sqrt[5]{11}) / \mathbf{Q}$ has discriminant $5^{5} 11^{4}$.

(iv) Since $J_{1} J_{2}=\mathbf{Q}\left(\mu_{25}\right) \mathbf{Q}\left(\mu_{11}\right)^{+}$it is clear that $\mathfrak{l}$ ramifies in $J_{2} / k$. We recall [W, Proposition 2.1] that $\mathbf{Q}\left(\mu_{25}\right)$ has discriminant $5^{35}$.

Remark 3.4 Another quick way to show that $J_{1} / k$ and $K_{1} / k$ are unramified above 5 is provided by Proposition 2.1 and the observation that our Kummer generators belong to $S\left(A_{1} \rightarrow A_{0} / k\right)$.

Lemma 3.5 (i) The 5-ray class field of $k$ with conductor $\mathfrak{p}_{1} \mathfrak{p}_{2} \mathfrak{p}_{3} \mathfrak{p}_{4}$ is $J_{1} K_{1}$. (ii) The 5-ray class field of $k$ with conductor $\mathfrak{l}^{2} \mathfrak{p}_{1} \mathfrak{p}_{2} \mathfrak{p}_{3} \mathfrak{p}_{4}$ is $J_{1} K_{1} K_{2}$.

Proof. We recall from [Coh, §3.2] a well known formula of class field theory

$$
[k(\mathfrak{m}): k]=\frac{h_{k} \phi(\mathfrak{m})}{\left[\mathfrak{o}_{k}^{*}: \mathfrak{o}_{k}^{*} \cap k_{\mathfrak{m}, 1}\right]} .
$$

In our case we know $h_{k}=1$ and $\mathfrak{o}_{k}^{*}$ is generated by $\pm \zeta, \phi$.

(i) For $\mathfrak{m}=\mathfrak{p}_{1} \mathfrak{p}_{2} \mathfrak{p}_{3} \mathfrak{p}_{4}$ we have $\phi(\mathfrak{m})=10^{4}$ and $\mathfrak{o}_{k}^{*} \cap k_{\mathfrak{m}, 1}$ generated by $\phi^{10}$. Thus the 5-ray class field has degree $5^{2}$, and so must equal $J_{1} K_{1}$.

(ii) For $\mathfrak{m}=\mathfrak{l}^{2} \mathfrak{p}_{1} \mathfrak{p}_{2} \mathfrak{p}_{3} \mathfrak{p}_{4}$ we have $\phi(\mathfrak{m})=20.10^{4}$ and $\mathfrak{o}_{k}^{*} \cap k_{\mathfrak{m}, 1}$ generated by $\phi^{20}$. Thus the 5 -ray class field has degree $5^{3}$, and so must equal $J_{1} K_{1} K_{2}$.

To end this section, we exhibit some (modular) units in the fields $K_{1}$ and $K_{2}$. Our descent calculations in $\S 6$ and $\S 7$ shall require further units in addition to these.

Lemma 3.6 (i) Let $\alpha=\sqrt[5]{\eta(1)}$ and $u_{i}=\varepsilon\left(\zeta^{i} \alpha\right)$. Then $u_{i}$ is a unit in $K_{1}=k(\alpha)$. The extension $J_{2} K_{1} / K_{1}$ has Kummer generator $u_{1} u_{2}^{2} u_{3}^{3} u_{4}^{4}$.

(i) Let $\beta=\sqrt[5]{11}$ and $u_{i}=\varepsilon\left(\zeta^{i} \beta\right)$. Then $u_{i}$ is a unit in $K_{2}=k(\beta)$. The extension $J_{1} K_{2} / K_{2}$ has Kummer generator $u_{1} u_{2}^{2} u_{3}^{3} u_{4}^{4}$. 
Proof. The cusps $\zeta \bar{\phi}, \zeta \phi$ have minimal polynomials

$$
f(x)=x^{4}+3 x^{3}+4 x^{2}+2 x+1, \quad g(x)=x^{4}-2 x^{3}+4 x^{2}-3 x+1 .
$$

We may check $\eta\left(\varepsilon(x)^{5}\right)=x f(x) / g(x)$.

(i) Each $u_{i}$ is a root of $x f(x)-g(x)=0$ and so is a unit. We apply Lemma 3.2 with $\tau=\alpha$ to give the stated Kummer generator.

(ii) Since $\eta(11)=-\phi^{15}$, each $u_{i}$ is a root of $x f(x)+\phi^{15} g(x)=0$ and so is a unit. We apply Lemma 3.2 with $\tau=\beta$ to give the stated Kummer generator.

\section{The Cassels-Tate pairing}

Let $C, D, \psi$ be as in the Introduction. There is an exact sequence

$$
0 \longrightarrow C[\psi] \longrightarrow C[m] \stackrel{\psi}{\longrightarrow} D[\widehat{\psi}] \longrightarrow 0 .
$$

Taking Galois cohomology and restricting to Selmer groups we obtain

$$
D[\widehat{\psi}](F) \longrightarrow S^{(\psi)}(C / F) \longrightarrow S^{(m)}(C / F) \longrightarrow S^{(\widehat{\psi})}(D / K) .
$$

Proposition 4.1 There is an alternating pairing

$$
S^{(\widehat{\psi})}(D / K) \times S^{(\widehat{\psi})}(D / K) \rightarrow \mathbf{Q} / \mathbf{Z}
$$

whose kernel is the image of $S^{(m)}(C / F)$.

Proof. See Cassels [Ca2] or Milne [Mi].

We recall the definition of the pairing in the case $m$ is odd. Our treatment follows that of McCallum [Mc]. For $(*)$ a global element or map, we write $(*)_{\mathfrak{p}}$ for the corresponding local object. The following commutative diagram is considered both in its own right and with $F$ replaced by $F_{\mathfrak{p}}$ for each prime $\mathfrak{p}$.

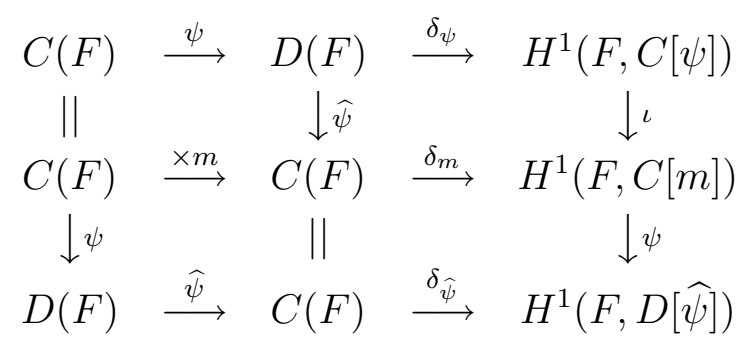


To define the pairing we take $x, y \in S^{(\widehat{\psi})}(D / F) \subset H^{1}(K, D[\widehat{\psi}])$ and suppose given $x_{1} \in H^{1}(F, C[m])$ lifting $x$. At each prime $\mathfrak{p}$ we choose $x_{\mathfrak{p}, 1} \in \operatorname{im} \delta_{m, \mathfrak{p}}$ such that $\psi\left(x_{\mathfrak{p}, 1}\right)=x_{\mathfrak{p}}$. Then $\psi\left(x_{\mathfrak{p}, 1}-x_{1, \mathfrak{p}}\right)=0$ and so there exists $\xi_{\mathfrak{p}} \in$ $H^{1}\left(F_{\mathfrak{p}}, C[\psi]\right)$ with $\iota\left(\xi_{\mathfrak{p}}\right)=x_{\mathfrak{p}, 1}-x_{1, \mathfrak{p}}$. We define

$$
\langle x, y\rangle=\sum_{\mathfrak{p}}\left(\xi_{\mathfrak{p}}, y_{\mathfrak{p}}\right)_{\mathfrak{p}}
$$

where $(\cdot, \cdot)_{\mathfrak{p}}$ is the Tate pairing $H^{1}\left(F_{\mathfrak{p}}, C[\psi]\right) \times H^{1}\left(F_{\mathfrak{p}}, D[\widehat{\psi}]\right) \rightarrow \mathbf{Q} / \mathbf{Z}$. Using Tate local duality and the product formula for the Tate pairing we may check that the definition is independent of all choices. It is clear that $\langle x, y\rangle=0$ whenever $x$ is in the image of $S^{(m)}(C / F)$.

Remark 4.2 More generally, the Cassels-Tate pairing is defined on the TateShafarevich group $\amalg(D / F)$. The restriction to $\amalg(D / F)[\widehat{\psi}]$ is the pairing induced by (10). We may implicit use of this fact in due course.

Remark 4.3 Suppose $C, D, \psi$ are defined over $F_{0} \subset F$. If $F$ is a normal extension of $F_{0}$ then (10) is $\operatorname{Gal}\left(F / F_{0}\right)$-equivariant. It is to be understood that the Galois action on $\mathbf{Q} / \mathbf{Z}$ is trivial.

We give a formula for (10) in the case where $\psi$ is the 5 -isogeny $A_{1} \rightarrow A_{0}$ and $F$ contains $K_{1}=\mathbf{Q}\left(A_{1}[5]\right)$. The exact sequence (9) becomes

$$
0 \longrightarrow A_{1}[\psi] \longrightarrow A_{1}[5] \stackrel{\psi}{\longrightarrow} A_{0}[\widehat{\psi}] \longrightarrow 0 .
$$

We choose a section for the map $\psi$ in (12) and use this to construct $x_{1}$ from $x$. As McCallum [Mc] observes we may now express (11) as a sum of local pairings

$$
\langle x, y\rangle=\sum_{\mathfrak{p}}\left\langle x_{\mathfrak{p}}, y_{\mathfrak{p}}\right\rangle_{\mathfrak{p}} .
$$

Furthermore the local pairing is trivial outside the usual set of bad primes, in our case those above 5 and 11. The description of $S\left(A_{0} \rightarrow A_{1} / F\right)$ given in Proposition 2.1 tells us that $x, y$ are already trivial at $\mathfrak{p} \mid 11$. So it only remains to compute the local pairing at $\mathfrak{p} \mid 5$.

In $\S 3$ we saw that $K_{1} / k$ has Kummer generator $\eta(1)=\left(1+\phi^{5}\right) /\left(1-\phi^{5}\right)$. We put $\alpha=\sqrt[5]{\eta(1)}$. By (8) the primes above 5 split in $K_{1} / k$. We label them $\mathfrak{L}_{0}, \mathfrak{L}_{1}, \ldots, \mathfrak{L}_{4}$ such that $\alpha \equiv \zeta^{i} \phi\left(\bmod \mathfrak{L}_{i}^{2}\right)$.

Lemma 4.4 Let $F$ be a number field with $F \supset K_{1}$. Let $\mathfrak{p} \mid 5$ be a prime and let $e=e(\mathfrak{p} / 5)$. Then there exists $i=i(\mathfrak{p})$ in $\mathbf{Z} / 5 \mathbf{Z}$ such that $\alpha \equiv \zeta^{i} \phi$ $\left(\bmod \mathfrak{p}^{e / 2}\right)$. 
Proof. We have $i=i(\mathfrak{p})$ if and only if $\mathfrak{p} \mid \mathfrak{L}_{i}$.

Proposition 4.5 Let $F$ be a number field with $F \supset K_{1}$. Then the CasselsTate pairing on $S\left(A_{0} \rightarrow A_{1} / F\right) \subset F^{*} / F^{* 5}$ is given, up to scalars, by

$$
\left\langle\theta, \theta^{\prime}\right\rangle=\sum_{\mathfrak{p} \mid 5} \operatorname{Ind}_{\zeta}\left(\theta, \theta^{\prime}\right)_{\mathfrak{p}}^{i(\mathfrak{p})}
$$

where $(\cdot, \cdot)_{\mathfrak{p}}$ is the Hilbert norm residue symbol.

Remark 4.6 To remove the qualifier "up to scalars" we must specify the isomorphism $A_{0}[\widehat{\psi}] \simeq \mu_{5}$ used to embed $S\left(A_{0} \rightarrow A_{1} / F\right)$ inside $F^{*} / F^{* 5}$. For the proof of Theorems 1 and 2, a formula "up to scalars" is good enough.

Lemma 4.7 Let $P \in A_{1}[\psi]$ and $Q \in A_{0}[\widehat{\psi}]$ with $e_{\psi}(P, Q)=\zeta$. Then we may label the inverse image $\psi^{-1}(Q)=\left\{Q_{0}, Q_{1}, \ldots, Q_{4}\right\}$ such that $Q_{i}$ belongs to the kernel of reduction $\bmod \mathfrak{L}_{i}$.

Proof. Let $\sim$ denote reduction mod $\mathfrak{L}_{i}$. By inspection of the Weierstrass equation (4) the reduction $\widetilde{A}_{1}\left(\mathbf{F}_{5}\right) \simeq \mathbf{Z} / 5 \mathbf{Z}$ is generated by $\widetilde{P}$. The kernel of the reduction map $A_{1}[5] \rightarrow \widetilde{A}_{1}\left(\mathbf{F}_{5}\right)$ is cyclic of order 5 . We choose a generator $Q_{i}$ with $\psi\left(Q_{i}\right)=Q$. Then $\operatorname{Gal}\left(K_{1} / k\right)$ permutes both the $\mathfrak{L}_{i}$ and the $Q_{i}$.

The Weil pairing and Hilbert's theorem 90 allow us to identify

$$
\begin{aligned}
H^{1}\left(F, A_{1}[\psi]\right) & =\operatorname{Hom}\left(A_{0}[\widehat{\psi}], F^{*} / F^{* 5}\right) \\
H^{1}\left(F, A_{1}[5]\right) & =\operatorname{Hom}\left(A_{1}[5], F^{*} / F^{* 5}\right) \\
H^{1}\left(F, A_{0}[\widehat{\psi}]\right) & =\operatorname{Hom}\left(A_{1}[\psi], F^{*} / F^{* 5}\right) .
\end{aligned}
$$

We give a more precise version of Proposition 4.5.

Lemma 4.8 Let $F$ be a number field with $F \supset K_{1}$. Let $P, Q_{i} \in A_{1}[5]$ be chosen as in Lemma 4.7. Then the Cassels-Tate pairing on $S\left(A_{0} \rightarrow A_{1} / F\right) \subset$ $\operatorname{Hom}\left(A_{1}[\psi], F^{*} / F^{* 5}\right)$ is given by

$$
\langle x, y\rangle=\sum_{\mathfrak{p} \mid 5} \operatorname{Ind}_{\zeta}\left(x\left(Q_{1}-Q_{0}\right), y(P)\right)_{\mathfrak{p}}^{i(\mathfrak{p})} .
$$

Proposition 4.5 follows immediately from Lemma 4.8, since $P$ and $Q_{1}-Q_{0}$ are both generators for $A_{1}[\psi]$. 
Lemma 4.9 Let $\mathfrak{p} \mid 5$ with $i=i(\mathfrak{p})$. Then the local connecting map $\delta_{5, \mathfrak{p}}: A_{1}\left(F_{\mathfrak{p}}\right) \rightarrow \operatorname{Hom}\left(A_{1}[5], F_{\mathfrak{p}}^{*} / F_{\mathfrak{p}}^{* 5}\right)$ has image

$$
\left\{x \in \operatorname{Hom}\left(A_{1}[5], \mathfrak{D}_{\mathfrak{p}}^{*} / \mathfrak{O}_{\mathfrak{p}}^{* 5}\right) \mid F_{\mathfrak{p}}\left(\sqrt[5]{x\left(Q_{i}\right)}\right) / F_{\mathfrak{p}} \text { is unramified }\right\}
$$

Proof. Let $x=\delta_{5, \mathfrak{p}}(T)$ for some $T \in A_{1}\left(F_{\mathfrak{p}}\right)$. The description of $\operatorname{im} \delta_{\widehat{\psi}, \mathfrak{p}}$ used in the proof of Proposition 2.1 shows that $x(P)$ is a unit. Let $T^{\prime} \in$ $A_{1}\left(\bar{F}_{\mathfrak{p}}\right)$ with $5 T^{\prime}=T$. Then $x$ is represented by the cocycle $\sigma\left(T^{\prime}\right)-T^{\prime}$ in $H^{1}\left(F_{\mathfrak{p}}, A_{1}[5]\right)$. But if $\sigma$ belongs to the inertia subgroup, then $\sigma\left(T^{\prime}\right)-T^{\prime}$ belongs to the kernel of reduction $\bmod \mathfrak{p}$ and $e_{5}\left(Q_{i}, \sigma\left(T^{\prime}\right)-T^{\prime}\right)=1$. Hence $x\left(Q_{i}\right)$ is unramified.

We have shown that $\operatorname{im} \delta_{5, \mathfrak{p}}$ belongs to (14). But im $\delta_{5, \mathfrak{p}} \subset H^{1}\left(F_{\mathfrak{p}}, A_{1}[5]\right)$ is a maximal isotropic subspace with respect to the Tate pairing. A counting argument completes the proof of the lemma.

The identifications (13) allow us to express the Tate pairing in terms of the Hilbert norm residue symbol.

Lemma 4.10 The Tate pairing $H^{1}\left(F_{\mathfrak{p}}, A_{1}[\psi]\right) \times H^{1}\left(F_{\mathfrak{p}}, A_{0}[\widehat{\psi}]\right) \rightarrow \mathbf{Q} / \mathbf{Z}$ is given by

$$
(x, y)_{\mathfrak{p}}=\operatorname{Ind}_{\zeta}(x(Q), y(P))_{\mathfrak{p}}^{-1}
$$

where $(\cdot, \cdot)_{\mathfrak{p}}$ on the right is the Hilbert norm residue symbol.

Proof. We recall $e_{\psi}(P, Q)=\zeta$. The lemma follows by a standard cup product calculation.

Proof of Lemma 4.8. The map $\psi$ in (12) has section $Q \mapsto Q_{0}$. Let $x, y$ belong to $S\left(A_{0} \rightarrow A_{1} / F\right)$. By (13) we view $x, y$ as maps $A_{1}[\psi] \rightarrow F^{*} / F^{* 5}$. Then $x_{1}: A_{1}[5] \rightarrow F^{*} / F^{* 5}$ extends $x$ via $Q_{0} \mapsto 1$. For each $\mathfrak{p} \mid 5$ we extend $x_{\mathfrak{p}}$ to $x_{\mathfrak{p}, 1}: A_{1}[5] \rightarrow F_{\mathfrak{p}}^{*} / F_{\mathfrak{p}}^{* 5}$ via $Q_{i(\mathfrak{p})} \mapsto 1$. Then $x_{\mathfrak{p}, 1} \in \operatorname{im} \delta_{5, \mathfrak{p}}$ and $\xi_{\mathfrak{p}}(Q)=$ $x\left(Q_{0}-Q_{i(\mathfrak{p})}\right)=x\left(Q_{1}-Q_{0}\right)^{-i(\mathfrak{p})}$. The local pairing is

$$
\begin{aligned}
\left\langle x_{\mathfrak{p}}, y_{\mathfrak{p}}\right\rangle_{\mathfrak{p}} & =\left(\xi_{\mathfrak{p}}, y_{\mathfrak{p}}\right)_{\mathfrak{p}} \\
& =\operatorname{Ind}_{\zeta}\left(\xi_{\mathfrak{p}}(Q), y_{\mathfrak{p}}(P)\right)_{\mathfrak{p}}^{-1} \\
& =\operatorname{Ind}_{\zeta}\left(x_{\mathfrak{p}}\left(Q_{1}-Q_{0}\right), y_{\mathfrak{p}}(P)\right)_{\mathfrak{p}}^{i(\mathfrak{p})}
\end{aligned}
$$

This completes the proof of Lemma 4.8 and so of Proposition 4.5. 


\section{Some Galois modules}

The polynomials $x^{5}+2 x^{4}+6 x^{3}-2 x^{2}+4 x-1$ and $x^{5}-11$ have splitting fields $K_{1}=\mathbf{Q}\left(A_{1}[5]\right)$ and $K_{2}=\mathbf{Q}\left(A_{2}[5]\right)$. In each case the Galois group is

$$
G:=\left\langle\sigma, \tau \mid \sigma^{4}=\tau^{5}=1, \sigma \tau \sigma^{-1}=\tau^{2}\right\rangle .
$$

In preparation for the proof of Theorems 1 and 2 , we give some preliminaries on $\mathbf{Z} / 5 \mathbf{Z}[G]$-modules. We define $\psi: G \rightarrow(\mathbf{Z} / 5 \mathbf{Z})^{*}$ via $\sigma \mapsto 2$ and $\tau \mapsto 1$. Any $\mathbf{Z} / 5 \mathbf{Z}[G]$-module $M$ may be decomposed into $\sigma$-eigenspaces

$$
M=M^{1} \oplus M^{\psi} \oplus M^{\psi^{2}} \oplus M^{\psi^{3}}
$$

where $M^{\chi}=\{x \in M \mid \sigma x=\chi(\sigma) x\}$. If $M=M^{\chi}$ we say that $M$ is described by $\chi$. In particular $\psi$ describes the action of $G$ on $\langle\tau\rangle$ via conjugation.

Lemma 5.1 Let $M$ be a $\mathbf{Z} / 5 \mathbf{Z}[G]$-module with $M /(\tau-1) M \simeq \mathbf{Z} / 5 \mathbf{Z}$ as an abelian group. Then

(i) $M /(\tau-1) M$ is described by some character $\chi: G \rightarrow(\mathbf{Z} / 5 \mathbf{Z})^{*}$.

(ii) $M$ has dimension $d:=\operatorname{dim}_{\mathbf{Z} / 5 \mathbf{Z}} M$ with $d \leq 5$.

(iii) The pair $(\chi, d)$ uniquely determines $M$ as a $G$-module.

(iv) If $d \leq 4$ then $\operatorname{End}_{G}(M) \simeq \mathbf{Z} / 5 \mathbf{Z}$.

Proof. (i) This is clear.

(ii) Let $M_{i}=(\tau-1)^{i} M$. The decreasing filtration of $\mathbf{Z} / 5 \mathbf{Z}[G]$-modules

$$
M=M_{0} \supset M_{1} \supset M_{2} \supset \ldots
$$

satisfies $\operatorname{dim}_{\mathbf{Z} / 5 \mathbf{Z}} M_{i} / M_{i+1} \geq \operatorname{dim}_{\mathbf{Z} / 5 \mathbf{Z}} M_{i+1} / M_{i+2}$. But $\operatorname{dim}_{\mathbf{Z} / 5 \mathbf{Z}} M_{0} / M_{1}=1$, so $d=\min \left\{i \mid M_{i}=0\right\}$. Since $(\tau-1)^{5} \equiv 0(\bmod 5)$ we must have $d \leq 5$.

(iii) We pick $x \in M^{\chi}$ a generator for $M /(\tau-1) M$. Then $M$ has basis $x,(\tau-1) x, \ldots,(\tau-1)^{d-1} x$ as a $\mathbf{Z} / 5 \mathbf{Z}$-vector space. The actions of $\sigma$ and $\tau$ on this basis are uniquely determined.

(iv) The quotient $M_{i} / M_{i+1}$ is described by $\chi \psi^{i}$. Thus for $d \leq 4$ the decomposition (15) is into 1-dimensional spaces, and the element $x$ in the proof of (iii) is uniquely determined up to scalars.

We write $M(\chi, d)$ for the $\mathbf{Z} / 5 \mathbf{Z}[G]$-module described in Lemma 5.1. We abbreviate $M(\chi)=M(\chi, 1)$. The filtration (16) becomes

$$
M(\chi, d) \supset M(\chi \psi, d-1) \supset \ldots \supset M\left(\chi \psi^{d-1}, 1\right) \supset 0 .
$$

For $M$ a $G$-module we recall that $M^{*}:=\operatorname{Hom}(M, \mathbf{Z} / 5 \mathbf{Z})$ is a $G$-module via $g \theta=\theta \cdot g^{-1}$. 
Lemma $5.2 M(\chi, d)^{*} \simeq M\left(\chi^{-1} \psi^{1-d}, d\right)$.

Proof. The case $d=1$ is clear. The general case follows from Lemma 5.1 and the observation $\operatorname{coker}\left(\tau-1 \mid M^{*}\right) \simeq \operatorname{ker}(\tau-1 \mid M)^{*}$.

With properties of the Cassels-Tate pairing in mind, we say that a bilinear form $\langle\rangle:, M \times N \rightarrow \mathbf{Q} / \mathbf{Z}$ is $G$-equivariant if $\langle g x, g y\rangle=\langle x, y\rangle$ for all $g \in G$. Equivalently $M \rightarrow N^{*}$ is a $G$-module homomorphism.

Lemma 5.3 Any non-zero G-equivariant pairing on $M(\chi, d)$ has odd rank. In particular there are no non-zero alternating $G$-equivariant pairings.

Proof. Suppose $f: M(\chi, d) \rightarrow M\left(\chi^{-1} \psi^{1-d}, d\right)$ is a $G$-module map of rank $r$. Then $\operatorname{im} f=M\left(\chi^{-1} \psi^{1-r}, r\right)$. We deduce $\chi=\chi^{-1} \psi^{1-r}$ and so $r$ is odd.

Lemma 5.4 Assume $d \leq 4$. Then any non-zero alternating $G$-equivariant pairing on $M:=M(\chi, d) \oplus M\left(\chi^{-1} \psi^{1-d}, d\right)$ is non-degenerate.

Proof. We claim that, up to scalars, $M$ admits a unique alternating $G$ equivariant pairing. By Lemma 5.3 any such pairing is trivial when restricted to either summand. We are reduced to showing that there is a unique $G$ equivariant bilinear form

$$
M(\chi, d) \times M\left(\chi^{-1} \psi^{1-d}, d\right) \rightarrow \mathbf{Z} / 5 \mathbf{Z} .
$$

Lemma 5.2 gives the existence. Lemma 5.1(iv) gives the uniqueness up to scalars. Finally we observe that the pairing constructed is non-degenerate.

We give an example typical of the $G$-modules we encounter. The group $G$ acts on the affine line $\mathbf{Z} / 5 \mathbf{Z}$ via $\sigma: x \mapsto 2 x$ and $\tau: x \mapsto x+1$. The corresponding permutation representation, with coefficients in $\mathbf{Z} / 5 \mathbf{Z}$, is $M(1,5)$. We construct further $G$-modules using $M\left(\chi_{1} \chi_{2}, d\right)=M\left(\chi_{1}\right) \otimes M\left(\chi_{2}, d\right)$.

\section{Descent calculations over $K_{1}=\mathbf{Q}\left(A_{1}[5]\right)$}

We recall that $K_{1} / k$ has Kummer generator $\eta(1)=\left(1+\phi^{5}\right) /\left(1-\phi^{5}\right)$. We put $\alpha=\sqrt[5]{\eta(1)}$. Then $\operatorname{Gal}\left(K_{1} / \mathbf{Q}\right) \simeq G$ via

$$
\begin{array}{ll}
\sigma(\zeta)=\zeta^{3} & \sigma(\alpha)=-1 / \alpha \\
\tau(\zeta)=\zeta & \tau(\alpha)=\zeta \alpha .
\end{array}
$$


The cyclotomic character $\omega$, and the character $\psi$ of $\S 5$ are related via $\psi=$ $\omega^{-1}$. The primes 5 and 11 factor in $K_{1}$ as

$$
(5)=\mathfrak{L}_{0}^{4} \mathfrak{L}_{1}^{4} \mathfrak{L}_{2}^{4} \mathfrak{L}_{3}^{4} \mathfrak{L}_{4}^{4} \quad(11)=\mathfrak{P}_{1}^{5} \mathfrak{P}_{2}^{5} \mathfrak{P}_{3}^{5} \mathfrak{P}_{4}^{5}
$$

with $\alpha \equiv \zeta^{i} \phi\left(\bmod \mathfrak{L}_{i}^{2}\right)$ and $\mathfrak{P}_{i} \mid \mathfrak{p}_{i}$. In $\S 3$ we saw that $H_{1}:=J_{1} K_{1}$ is the 5 -ray class field of $k$ for conductor $(11)=\mathfrak{p}_{1} \mathfrak{p}_{2} \mathfrak{p}_{3} \mathfrak{p}_{4}$.

Lemma 6.1 The extension $H_{1} / K_{1}$ is unramified at all primes. Furthermore the primes above 5 and 11 are inert in this extension.

Proof. Only primes above 11 ramify in $H_{1} / k$. By considering suitable ratios of our Kummer generators for $J_{1} / k$ and $K_{1} / k$ we see that $\mathfrak{p}_{i}$ cannot be totally ramified in $H_{1} / k$. Hence $H_{1} / K_{1}$ is unramified as claimed. Now $H_{1} / K_{1}$ is a translate of $\mathbf{Q}\left(\mu_{11}\right)^{+} / \mathbf{Q}$. Since 5 is inert in $\mathbf{Q}\left(\mu_{11}\right)^{+} / \mathbf{Q}$ and $\mathfrak{L}_{i}$ has residue field $\mathbf{F}_{5}$, it follows that $\mathfrak{L}_{i}$ is inert. Finally the definitions of $J_{1}$ and $K_{1}$ give $\# A_{1}\left(H_{1}\right)(5) \geq 5^{3}$, so by Lemma 1.7 the $\mathfrak{P}_{i}$ are inert.

Proposition 6.2 The 5-class group of $K_{1}$ is $\mathfrak{C l}_{K_{1}}(5) \simeq \mathbf{Z} / 5 \mathbf{Z}$. It is generated by any prime above 5 or 11 .

Proof. Let $B=\mathfrak{C l}_{K_{1}}(5)$. By Lemma 3.5(i) we know that $H_{1}$ is the maximal unramified 5-extension of $K_{1}$ which is abelian over $k$. Thus

$$
B /(\tau-1) B \simeq \operatorname{Gal}\left(H_{1} / K_{1}\right) \simeq \mathbf{Z} / 5 \mathbf{Z}
$$

as abelian groups. By Lemma $6.1, \mathfrak{P}_{1}$ is inert in $H_{1} / K_{1}$ and so generates $B /(\tau-1) B$. Since $(\tau-1)^{5} \subset 5 \mathbf{Z}_{5}[\tau]$ it follows that $\mathfrak{P}_{1}$ generates $B$ as a $\mathbf{Z}_{5}[\tau]$-module. But $\tau\left(\mathfrak{P}_{1}\right)=\mathfrak{P}_{1}$ and $\mathfrak{P}_{1}^{5}=\mathfrak{p}_{1}$ is principal. Thus $B \simeq \mathbf{Z} / 5 \mathbf{Z}$ as claimed. By Lemma 6.1, $B$ is generated by any prime above 5 or 11 .

Remark 6.3 Since $\sigma\left(\mathfrak{L}_{0}\right)=\mathfrak{L}_{0}$ and $\tau\left(\mathfrak{P}_{1}\right)=\mathfrak{P}_{1}$ the action of $G$ on $\mathfrak{C l}_{K_{1}}(5)$ is trivial. In particular $\mathfrak{P}_{1} \sim \mathfrak{P}_{2} \sim \mathfrak{P}_{3} \sim \mathfrak{P}_{4}$.

We turn our attention to the units in $K_{1}$. We write

$$
u_{i}=\frac{\phi \zeta^{i} \alpha+1}{\zeta^{i} \alpha-\phi} \quad v_{i}=\frac{\zeta^{i} \alpha+1}{\zeta^{i} \alpha-1} .
$$


The $u_{i}$ are units by Lemma 3.6. For the $v_{i}$ we have $\left(v_{i}+1\right)^{5} /\left(v_{i}-1\right)^{5}=$ $\left(1+\phi^{5}\right) /\left(1-\phi^{5}\right)$. Thus each $v_{i}$ is a root of

$$
\phi^{5}\left(x^{5}+10 x^{3}+5 x\right)-\left(5 x^{4}+10 x^{2}+1\right)=0
$$

and so is a unit. It is easy to check

$$
\begin{array}{ll}
\sigma\left(u_{i}\right)=u_{2 i} & \sigma\left(v_{i}\right)=-1 / v_{2 i} \\
\tau\left(u_{i}\right)=u_{i+1} & \tau\left(v_{i}\right)=v_{i+1}
\end{array}
$$

and we have relations $\prod u_{i}=1, \prod v_{i}=\phi^{-5}$. Thus the subgroups of $K_{1}^{*} / K_{1}^{* 5}$ generated by $u_{1}, u_{2}, u_{3}, u_{4}$ and $v_{1}, v_{2}, v_{3}, v_{4}$ are quotients of the $G$-modules $M(1,4)$ and $M\left(\omega^{2}, 4\right)$.

Proposition 6.4 The units $\zeta, \phi, u_{1}, u_{2}, u_{3}, u_{4}, v_{1}, v_{2}, v_{3}, v_{4}$ generate a subgroup of $\mathfrak{O}_{K_{1}}^{*}$ of index prime to 5 .

Proof. By Dirichlet it suffices to check that the elements listed are independent in $K_{1}^{*} / K_{1}^{* 5}$. There is a $G$-module homomorphism

$$
M:=M(\omega) \oplus M\left(\omega^{2}\right) \oplus M(1,4) \oplus M\left(\omega^{2}, 4\right) \rightarrow K_{1}^{*} / K_{1}^{* 5}
$$

where the summands correspond to $\zeta, \phi,\left\langle u_{1}, u_{2}, u_{3}, u_{4}\right\rangle$ and $\left\langle v_{1}, v_{2}, v_{3}, v_{4}\right\rangle$. We suppose for a contradiction that this map has non-trivial kernel. Then this kernel meets

$$
\begin{aligned}
\operatorname{ker}(\tau-1 \mid M) & =M(\omega) \oplus M\left(\omega^{2}\right) \oplus M(\omega) \oplus M\left(\omega^{3}\right) \\
& =\left\langle\zeta, \phi, u_{1} u_{2}^{2} u_{3}^{3} u_{4}^{4}, v_{1} v_{2}^{2} v_{3}^{3} v_{4}^{4}\right\rangle .
\end{aligned}
$$

Dividing into $\sigma$-eigenspaces we learn that one of the elements

$$
\zeta^{i}\left(u_{1} u_{2}^{2} u_{3}^{3} u_{4}^{4}\right)^{j}, \quad \phi, \quad v_{1} v_{2}^{2} v_{3}^{3} v_{4}^{4}
$$

is a 5 th power. For $\zeta$ and $\phi$ this is clearly false, since no unit can be a Kummer generator for $K_{1} / k$. The smallest prime to split completely in $K_{1} / \mathbf{Q}$ is $p=101$. Reducing modulo primes above $p$ we obtain a contradiction.

Remark 6.5 Further to the proof Proposition 6.4, a brutal computer calculation shows

$$
\begin{array}{ll}
\pi_{1} \pi_{2}^{3} \pi_{3}^{2} \pi_{4}^{4} \equiv\left(u_{1} u_{2}^{2} u_{3}^{3} u_{4}^{4}\right)^{3} \quad\left(\bmod K_{1}^{* 5}\right) \\
\pi_{1} \pi_{2}^{2} \pi_{3}^{3} \pi_{4}^{4} \equiv\left(v_{1} v_{2}^{2} v_{3}^{3} v_{4}^{4}\right)^{3} \quad\left(\bmod K_{1}^{* 5}\right)
\end{array}
$$


Remark 6.6 According to pari the field $K_{1}$ has class number 5, and

$$
\phi, u_{1}, u_{2}, u_{3}, u_{4}, v_{1}, v_{2}, v_{3}, v_{4}
$$

is a set of fundamental units. However pari assumes the Generalised Riemann Hypothesis, whereas our results are unconditional.

We apply Propositions 2.1 and 2.2 in the case $F=K_{1}$.

Proposition 6.7 The Selmer groups attached to the 5-isogenies (1) are

$$
\begin{array}{ll}
S\left(A_{0} \rightarrow A_{1} / K_{1}\right) \simeq(\mathbf{Z} / 5 \mathbf{Z})^{8} & S\left(A_{2} \rightarrow A_{0} / K_{1}\right) \simeq(\mathbf{Z} / 5 \mathbf{Z})^{14} \\
S\left(A_{1} \rightarrow A_{0} / K_{1}\right) \simeq(\mathbf{Z} / 5 \mathbf{Z})^{2} & S\left(A_{0} \rightarrow A_{2} / K_{1}\right)=0 .
\end{array}
$$

Proof. By Propositions 6.2 and 6.4 the space

$$
\left\{\theta \in K_{1}^{*} / K_{1}^{* 5} \mid \operatorname{ord}_{\mathfrak{p}}(\theta) \equiv 0(\bmod 5) \text { for all } \mathfrak{p}\right\}
$$

has basis $\zeta, \phi, u_{1}, u_{2}, u_{3}, u_{4}, v_{1}, v_{2}, v_{3}, v_{4}, 11$. Here 11 is a contribution from the class group or "virtual unit". We choose characters $\left(\mathfrak{O}_{K_{1}} / \mathfrak{P}_{i}\right)^{*} \rightarrow \mathbf{Z} / 5 \mathbf{Z}$ and compute these characters on our basis. Notice that by inspection of our Kummer generator for $K_{1} / k$ we have $11 \equiv\left(1+\phi^{5}\right)^{2} \equiv\left(1-\phi^{5}\right)^{2}\left(\bmod K_{1}^{* 5}\right)$. Thus our table is easily computed by hand.

\begin{tabular}{c|cccc} 
& $\mathfrak{P}_{1}$ & $\mathfrak{P}_{2}$ & $\mathfrak{P}_{3}$ & $\mathfrak{P}_{4}$ \\
\hline$\zeta$ & 1 & 3 & 2 & 4 \\
$\phi$ & 2 & 3 & 3 & 2 \\
$u_{i}$ & 2 & 2 & 2 & 2 \\
$v_{i}$ & 0 & 0 & 0 & 0 \\
11 & 2 & 2 & 2 & 2
\end{tabular}

By Proposition 2.1 we deduce

$$
S\left(A_{0} \rightarrow A_{1} / K_{1}\right) \simeq\left\langle u_{1}^{\prime}, u_{2}^{\prime}, u_{3}^{\prime}, u_{4}^{\prime}, v_{1}, v_{2}, v_{3}, v_{4}\right\rangle \subset K_{1}^{*} / K_{1}^{* 5}
$$

where $u_{i}^{\prime}=u_{i} / 11$. Propositions 2.1 and 6.2 show that $S\left(A_{0} \rightarrow A_{2} / K_{1}\right)$ is trivial. The remaining statements follow by Proposition 2.2.

Proposition 6.7 furnishes the estimate rank $A\left(K_{1}\right) \leq 8$. We improve on this by computing the Cassels-Tate pairing

$$
S\left(A_{0} \rightarrow A_{1} / K_{1}\right) \times S\left(A_{0} \rightarrow A_{1} / K_{1}\right) \rightarrow \mathbf{Q} / \mathbf{Z} .
$$


As a $G$-module we have

$$
S\left(A_{0} \rightarrow A_{1} / K_{1}\right) \simeq M(1,4) \oplus M\left(\omega^{2}, 4\right)
$$

where the summands correspond to $\left\langle u_{1}^{\prime}, u_{2}^{\prime}, u_{3}^{\prime}, u_{4}^{\prime}\right\rangle$ and $\left\langle v_{1}, v_{2}, v_{3}, v_{4}\right\rangle$. By Lemma 5.3 the pairing (17) is trivial when restricted to either summand. It therefore suffices for us to compute the entries $\left\langle u_{r}^{\prime}, v_{s}\right\rangle$. By Proposition 4.5 and the action of $\operatorname{Gal}\left(K_{1} / k\right)$ we have

$$
\begin{aligned}
\left\langle u_{r}^{\prime}, v_{s}\right\rangle & =\sum_{i=0}^{4} \operatorname{Ind}_{\zeta}\left(u_{r}^{\prime}, v_{s}\right)_{\mathfrak{L}_{i}}^{i} \\
& =\sum_{i=0}^{4} \operatorname{Ind}_{\zeta}\left(u_{r-i}^{\prime}, v_{s-i}\right)_{\mathfrak{L}_{0}}^{i}
\end{aligned}
$$

We recall that $\mathfrak{L}_{0}$ is the prime of $K_{1}$ above 5 such that $\alpha \equiv \phi\left(\bmod \mathfrak{L}_{0}^{2}\right)$. But $\alpha$ is a 5 th root of

$$
\eta(1)=\frac{1+\phi^{5}}{1-\phi^{5}}=-\phi^{15}\left(1+\frac{10\left(\overline{\phi^{5}}-\phi^{5}\right)}{1+10 \phi^{5}}\right) .
$$

The binomial theorem gives $\alpha \equiv-\phi^{3}\left(\bmod \mathfrak{L}_{0}^{6}\right)$ and for $r \neq 0$ it follows

$$
\begin{array}{ll}
u_{r} \equiv\left(\zeta^{r} \phi^{4}-1\right) /\left(\zeta^{r} \phi^{3}+\phi\right) & \left(\bmod \mathfrak{L}_{0}^{6}\right) \\
v_{s} \equiv\left(\zeta^{s} \phi^{3}-1\right) /\left(\zeta^{s} \phi^{3}+1\right) & \left(\bmod \mathfrak{L}_{0}^{6}\right) .
\end{array}
$$

\begin{tabular}{|c|c|c|c|c|c|c|c|c|c|c|c|}
\hline,$\quad)_{\mathfrak{L}_{0}}$ & $v_{0}$ & $v_{1}$ & $v_{2}$ & $v_{3}$ & $v_{4}$ & $5\langle\rangle$, & $v_{0}$ & $v_{1}$ & $v_{2}$ & $v_{3}$ & $v_{4}$ \\
\hline$\overline{u_{0}^{\prime}}$ & 1 & $\zeta^{2}$ & $\zeta^{4}$ & $\zeta$ & $\zeta^{3}$ & $u_{0}^{\prime}$ & 0 & 4 & 1 & 1 & 4 \\
\hline$u_{1}^{\prime}$ & $\zeta^{3}$ & $\zeta^{2}$ & $\zeta^{4}$ & 1 & $\zeta$ & $u_{1}^{\prime}$ & 4 & 0 & 4 & 1 & 1 \\
\hline$u_{2}^{\prime}$ & $\zeta$ & 1 & $\zeta^{4}$ & $\zeta^{2}$ & $\zeta^{3}$ & $u_{2}^{\prime}$ & 1 & 4 & 0 & 4 & 1 \\
\hline$u_{3}^{\prime}$ & $\zeta^{4}$ & $\zeta^{2}$ & $\zeta^{3}$ & $\zeta$ & 1 & $u_{3}^{\prime}$ & 1 & 1 & 4 & 0 & 4 \\
\hline$u_{4}^{\prime}$ & $\zeta^{2}$ & $\zeta^{4}$ & 1 & $\zeta$ & $\zeta^{3}$ & $u_{4}^{\prime}$ & 4 & 1 & 1 & 4 & 0 \\
\hline
\end{tabular}

Using these approximations we are reduced to computing the Hilbert norm residue symbol at the prime $\mathfrak{l}=(1-\zeta)$ of $k=\mathbf{Q}\left(\mu_{5}\right)$. This is straightforward, if tedious, to do by hand. See [CF, Exercises 1 and 2]. We find

The matrix on the right has rank 3, and so the pairing (17) has rank 6 . The kernel is generated by $u_{1} u_{2}^{2} u_{3}^{3} u_{4}^{4}$ and $v_{1} v_{2}^{2} v_{3}^{3} v_{4}^{4}$. By Corollary 1.6 and Lemma 3.6 the first of these elements is accounted for by torsion, whereas the second is not.

By Propositions 4.1 and 6.7 we deduce

$$
S\left(A_{1} \stackrel{\times 5}{\rightarrow} A_{1} / K_{1}\right) \simeq(\mathbf{Z} / 5 \mathbf{Z})^{4} .
$$


This furnishes the estimate $\operatorname{rank} A\left(K_{1}\right) \leq 2$. Since $\operatorname{rank} A(k)=0$ the action of $\operatorname{Gal}\left(K_{1} / k\right)$ on $A\left(K_{1}\right) \otimes \mathbf{Q}$ forces $\operatorname{rank} A\left(K_{1}\right) \equiv 0(\bmod 4)$. Thus $\operatorname{rank} A\left(K_{1}\right)=0$ and $\amalg\left(A_{1} / K_{1}\right)[5] \simeq(\mathbf{Z} / 5 \mathbf{Z})^{2}$. However, to identify the 5primary part of the Tate-Shafarevich group we must work harder.

We aim to compute $S\left(A_{0} \stackrel{\times 5}{\rightarrow} A_{0} / K_{1}\right)$ as a $G$-module. To identify it as an abelian group we make use of the exact sequences

$$
\begin{aligned}
& 0 \longrightarrow S\left(A_{0} \rightarrow A_{1} / K_{1}\right) \longrightarrow S\left(A_{0} \stackrel{\times 5}{\longrightarrow} A_{0} / K_{1}\right) \stackrel{\psi_{1}}{\longrightarrow} S\left(A_{1} \rightarrow A_{0} / K_{1}\right) \\
& 0 \longrightarrow S\left(A_{0} \rightarrow A_{2} / K_{1}\right) \longrightarrow S\left(A_{0} \stackrel{\times 5}{\longrightarrow} A_{0} / K_{1}\right) \stackrel{\psi_{2}}{\longrightarrow} S\left(A_{2} \rightarrow A_{0} / K_{1}\right)
\end{aligned}
$$

The images of the maps $\psi_{1}$ and $\psi_{2}$ are the kernels of the Cassels-Tate pairings

$$
\begin{aligned}
& \Psi_{1}: S\left(A_{1} \rightarrow A_{0} / K_{1}\right) \times S\left(A_{1} \rightarrow A_{0} / K_{1}\right) \rightarrow \mathbf{Z} / 5 \mathbf{Z} \\
& \Psi_{2}: S\left(A_{2} \rightarrow A_{0} / K_{1}\right) \times S\left(A_{2} \rightarrow A_{0} / K_{1}\right) \rightarrow \mathbf{Z} / 5 \mathbf{Z}
\end{aligned}
$$

Lemma 6.8 The pairings $\Psi_{1}$ and $\Psi_{2}$ have ranks 0 and 4 respectively.

Proof. The alternating pairing $\Psi_{1}$ is defined on $S\left(A_{1} \rightarrow A_{0} / K_{1}\right) \simeq(\mathbf{Z} / 5 \mathbf{Z})^{2}$. By Corollary 1.6 this Selmer group contains a contribution from torsion. Hence $\Psi_{1}$ is trivial. The exact sequences (19), together with Propositions 4.1 and 6.7 , now tell us that $S\left(A_{0} \stackrel{\times 5}{\rightarrow} A_{0} / K_{1}\right) \simeq(\mathbf{Z} / 5 \mathbf{Z})^{10}$ and that the pairing $\Psi_{2}$ has rank 4 .

The exact sequences (19) provide inclusions

$$
S\left(A_{0} \rightarrow A_{1} / K_{1}\right) \subset S\left(A_{0} \stackrel{\times 5}{\rightarrow} A_{0} / K_{1}\right) \subset S\left(A_{2} \rightarrow A_{0} / K_{1}\right) \subset K_{1}^{*} / K_{1}^{* 5}
$$

By Remark 6.5 there exist $w_{1}, w_{2}$ in $K_{1}$ with

$$
\begin{aligned}
& w_{1}^{5}=\pi_{1} \pi_{2}^{-2} \pi_{3}^{2} \pi_{4}^{-1}\left(u_{1} u_{2}^{2} u_{3}^{3} u_{4}^{4}\right)^{2} \\
& w_{2}^{5}=\pi_{1} \pi_{2}^{2} \pi_{3}^{-2} \pi_{4}^{-1}\left(v_{1} v_{2}^{2} v_{3}^{3} v_{4}^{4}\right)^{2} .
\end{aligned}
$$

A rather tedious calculation suggests we write $x_{1}=w_{1} u_{2}^{2} u_{3} u_{4}^{3}\left(v_{1} v_{2}^{2} v_{3}^{3} v_{4}^{4}\right)^{3}$ and $x_{2}=w_{2} v_{1}^{3} v_{2}^{3}\left(u_{1} u_{2}^{2} u_{3}^{3} u_{4}^{4}\right)^{3}$, whereupon, multiplying $x_{2}$ by a 5 th root of unity if necessary

$$
\begin{array}{lllr}
\sigma\left(x_{1}\right) & \equiv x_{1}^{3} \quad\left(\bmod K_{1}^{* 5}\right) & \sigma\left(x_{2}\right) \equiv x_{2}^{2} & \left(\bmod K_{1}^{* 5}\right) \\
\tau\left(x_{1}\right) \equiv x_{1} u_{2}^{3} u_{3} u_{4}^{3}\left(\bmod K_{1}^{* 5}\right) & \tau\left(x_{2}\right) \equiv x_{2} \phi^{2} v_{2}^{3} v_{3} v_{4}^{3}\left(\bmod K_{1}^{* 5}\right)
\end{array}
$$


Proposition 6.9 Multiplying $x_{1}$ by a 5th root of unity if necessary, the Selmer groups (21) are

$$
\begin{aligned}
& S\left(A_{0} \rightarrow A_{1} / K_{1}\right) \simeq\left\langle u_{1}^{\prime}, u_{2}^{\prime}, u_{3}^{\prime}, u_{4}^{\prime}, v_{1}, v_{2}, v_{3}, v_{4}\right\rangle \\
& S\left(A_{0} \stackrel{\times 5}{\rightarrow} A_{0} / K_{1}\right) \simeq\left\langle u_{1}, u_{2}, u_{3}, u_{4}, v_{1}, v_{2}, v_{3}, v_{4}, 11, x_{1}\right\rangle \\
& S\left(A_{2} \rightarrow A_{0} / K_{1}\right) \simeq\left\langle\zeta, \phi, u_{1}, u_{2}, u_{3}, u_{4}, v_{1}, v_{2}, v_{3}, v_{4}, 11, \alpha, x_{1}, x_{2}\right\rangle .
\end{aligned}
$$

Proof. The descriptions of $S\left(A_{0} \rightarrow A_{1} / K_{1}\right)$ and $S\left(A_{2} \rightarrow A_{0} / K_{1}\right)$ follow from Proposition 2.1. Now $S\left(A_{0} \stackrel{\times 5}{\longrightarrow} A_{0} / K_{1}\right)$ is the kernel of the pairing $\Psi_{2}$ and this pairing induces a pairing on the quotient

$$
\frac{S\left(A_{2} \rightarrow A_{0} / K_{1}\right)}{S\left(A_{0} \rightarrow A_{1} / K_{1}\right)} \simeq\left\langle\zeta, \phi, 11, \alpha, x_{1}, x_{2}\right\rangle .
$$

Decomposing into $\sigma$-eigenspaces, we learn that $S\left(A_{0} \stackrel{\times 5}{\rightarrow} A_{0} / K_{1}\right)$ has basis

$$
u_{1}, u_{2}, u_{3}, u_{4}, v_{1}, v_{2}, v_{3}, v_{4}, 11, z
$$

for some $z=\zeta^{i} x_{1}^{j}$. Since we consider ourselves free to multiply $x_{1}$ by a 5 th root of unity, it only remains to show $\zeta \notin S\left(A_{0} \stackrel{\times 5}{\rightarrow} A_{0} / K_{1}\right)$. To do this we identify (23) as a $G$-module. By (22)

$$
\begin{array}{ll}
\tau\left(x_{1}\right) \equiv x_{1} 11^{2} & \bmod \left\langle u_{1}^{\prime}, u_{2}^{\prime}, u_{3}^{\prime}, u_{4}^{\prime}, K_{1}^{* 5}\right\rangle \\
\tau\left(x_{2}\right) \equiv x_{2} \phi^{2} & \bmod \left\langle v_{1}, v_{2}, v_{3}, v_{4}, K_{1}^{* 5}\right\rangle
\end{array}
$$

whereas $\tau(\alpha)=\zeta \alpha$ and $\zeta, \phi, 11 \in k$ are fixed by $\tau$. Thus

$$
\frac{S\left(A_{2} \rightarrow A_{0} / K_{1}\right)}{S\left(A_{0} \rightarrow A_{1} / K_{1}\right)} \simeq M(\omega, 2) \oplus M\left(\omega^{2}, 2\right) \oplus M\left(\omega^{3}, 2\right) .
$$

By Lemma 5.4 the pairing $\Psi_{2}$ restricted to

$$
M\left(\omega^{2}, 2\right) \oplus M\left(\omega^{3}, 2\right) \simeq\left\langle\zeta, \phi, \alpha, x_{2}\right\rangle
$$

is either zero or non-degenerate. But it cannot be zero by our earlier consideration of $\sigma$-eigenspaces. Thus $\zeta \notin S\left(A_{0} \stackrel{\times 5}{\longrightarrow} A_{0} / K_{1}\right)$ and we are done.

Remark 6.10 An alternative proof of Proposition 6.9 is given by computing $\Psi_{2}$ via the formula of $[\mathrm{F} 0, \S 7.3]$. 
As a $G$-module we have

$$
S\left(A_{0} \stackrel{\times 5}{\rightarrow} A_{0} / K_{1}\right) \simeq M(1) \oplus M(\omega, 5) \oplus M\left(\omega^{2}, 4\right)
$$

where the summands correspond to $11,\left\langle u_{1}, u_{2}, u_{3}, u_{4}, x_{1}\right\rangle$ and $\left\langle v_{1}, v_{2}, v_{3}, v_{4}\right\rangle$. The contributions from torsion are 11 and $u_{1} u_{2}^{2} u_{3}^{3} u_{4}^{4}$. Thus

$$
\frac{S\left(A_{0} \stackrel{\times 5}{\rightarrow} A_{0} / K_{1}\right)}{\operatorname{im}\left(\delta \mid A_{0}[5]\right)} \simeq M(\omega, 4) \oplus M\left(\omega^{2}, 4\right) .
$$

Our earlier calculation of the pairing (17) shows that the Cassels-Tate pairing on (24) is non-zero. By Lemma 5.4 the Cassels-Tate pairing on (24) is nondegenerate. It follows that $\amalg\left(A_{0} / K_{1}\right)(5) \simeq(\mathbf{Z} / 5 \mathbf{Z})^{8}$.

Earlier we saw $\amalg\left(A_{1} / K_{1}\right)[5] \simeq(\mathbf{Z} / 5 \mathbf{Z})^{2}$. We claim that $\amalg\left(A_{1} / K_{1}\right)$ contains no element of order 25 . Indeed, from the exact sequences

$$
\begin{gathered}
A_{1}\left(K_{1}\right) \stackrel{\psi}{\longrightarrow} A_{0}\left(K_{1}\right) \longrightarrow S\left(A_{1} \rightarrow A_{0} / K_{1}\right) \longrightarrow \amalg\left(A_{1} / K_{1}\right)[\psi] \longrightarrow 0 \\
0 \longrightarrow \amalg\left(A_{1} / K_{1}\right)[\psi] \longrightarrow \amalg\left(A_{1} / K_{1}\right)(5) \stackrel{\psi}{\longrightarrow} \amalg\left(A_{0} / K_{1}\right)(5)
\end{gathered}
$$

we learn that $\amalg\left(A_{1} / K_{1}\right)(5)$ is finite and contains no copy of $(\mathbf{Z} / 25 \mathbf{Z})^{2}$. Our claim is now a well known consequence of the Cassels-Tate pairing.

Finally the exact sequence

$$
S\left(A_{2} \rightarrow A_{0} / K_{1}\right) \longrightarrow S\left(A_{2} \stackrel{\times 5}{\longrightarrow} A_{2} / K_{1}\right) \longrightarrow S\left(A_{0} \rightarrow A_{2} / K_{1}\right)=0
$$

shows that $S\left(A_{2} \stackrel{\times 5}{\rightarrow} A_{2} / K_{1}\right) \simeq(\mathbf{Z} / 5 \mathbf{Z})^{13}$. By Lemma 6.8 the Cassels-Tate pairing on this Selmer group has rank 4. Since the multiplication by 5 map on $A_{2}$ factors through $A_{0}$ and $\amalg\left(A_{0} / K_{1}\right)(5)$ is 5 -torsion, we deduce

$$
\amalg\left(A_{2} / K_{1}\right)(5) \simeq(\mathbf{Z} / 5 \mathbf{Z})^{4} \oplus(\mathbf{Z} / 25 \mathbf{Z})^{8} .
$$

This completes the proof of Theorem 1 .

\section{Descent calculations over $K_{2}=\mathbf{Q}\left(A_{2}[5]\right)$}

We recall that $K_{2} / k$ has Kummer generator 11 . We put $\beta=\sqrt[5]{11}$. Then $\operatorname{Gal}\left(K_{2} / \mathbf{Q}\right) \simeq G$ via

$$
\begin{array}{ll}
\sigma(\zeta)=\zeta^{2} & \sigma(\beta)=\beta \\
\tau(\zeta)=\zeta & \tau(\beta)=\zeta \beta .
\end{array}
$$


The cyclotomic character $\omega$ and the character $\psi$ of $\S 5$ are equal. The primes above 5 and 11 ramify in $K_{2} / k$. We write

$$
(5)=\mathfrak{L}^{20} \quad(11)=\mathfrak{P}_{1}^{5} \mathfrak{P}_{2}^{5} \mathfrak{P}_{3}^{5} \mathfrak{P}_{4}^{5}
$$

with $\mathfrak{P}_{i} \mid \mathfrak{p}_{i}$. In $\S 3$ we saw that $H_{2}:=J_{1} K_{1} K_{2}$ is the 5-ray class field of $k$ for conductor $\mathfrak{l}^{2} \mathfrak{p}_{1} \mathfrak{p}_{2} \mathfrak{p}_{3} \mathfrak{p}_{4}$.

Lemma 7.1 The extension $\mathrm{H}_{2} / K_{2}$ is unramified at all primes.

(i) The primes above 5 are inert in $J_{1} K_{2} / K_{2}$ and split in $K_{1} K_{2} / K_{2}$.

(ii) The primes above 11 are split in $J_{1} K_{2} / K_{2}$ and inert in $K_{1} K_{2} / K_{2}$.

Proof. Only primes above 11 ramify in $J_{1} / k$ and $K_{1} / k$. By considering ratios of Kummer generators we see that the extensions $J_{1} K_{2} / K_{2}$ and $K_{1} K_{2} / K_{2}$ are unramified. Hence the composite $H_{2} / K_{2}$ is unramified.

(i) The extensions $J_{1} K_{2} / K_{2}$ and $K_{1} K_{2} / K_{2}$ are translates of $\mathbf{Q}\left(\mu_{11}\right)^{+} / \mathbf{Q}$ and $K_{1} / k$. Since 5 is totally ramified in $K_{2} / \mathrm{Q}$ our claims follow.

(ii) The Kummer generator for $J_{1} K_{2} / K_{2}$ belongs to $S\left(A_{0} \rightarrow A_{2} / K_{2}\right)$. By Proposition 2.1 the primes above 11 split in $J_{1} K_{2} / K_{2}$. By Lemma 6.1 the primes above 11 cannot split completely in $H_{2} / K_{2}$. They are therefore inert in $K_{1} K_{2} / K_{2}$.

Proposition 7.2 The 5-class group of $K_{2}$ is $\mathfrak{C l}_{K_{2}}(5) \simeq(\mathbf{Z} / 5 \mathbf{Z})^{2}$. It is generated by the primes above 5 and 11 .

Proof. Let $B=\mathfrak{C l}_{K_{2}}(5)$. By Lemma 3.5(ii) we know that $H_{2}$ is the maximal unramified 5-extension of $K_{2}$ which is abelian over $k$. Thus

$$
B /(\tau-1) B \simeq \operatorname{Gal}\left(H_{2} / K_{2}\right) \simeq(\mathbf{Z} / 5 \mathbf{Z})^{2}
$$

as abelian groups. By Lemma 7.1, the primes $\mathfrak{P}_{1}$ and $\mathfrak{L}$ generate $B /(\tau-1) B$. Since $(\tau-1)^{5} \subset 5 \mathbf{Z}_{5}[\tau]$ it follows that $\mathfrak{P}_{1}$ and $\mathfrak{L}$ generate $B$ as a $\mathbf{Z}_{5}[\tau]$ module. But $\tau\left(\mathfrak{P}_{1}\right)=\mathfrak{P}_{1}, \tau(\mathfrak{L})=\mathfrak{L}$ and $\mathfrak{P}_{1}^{5}=\mathfrak{p}_{1}, \mathfrak{L}^{5}=\mathfrak{l}$ are principal. Thus $B \simeq(\mathbf{Z} / 5 \mathbf{Z})^{2}$ as claimed.

Remark 7.3 The action of $\tau$ on $\mathfrak{C l}_{K_{2}}(5)$ is trivial. By Lemma 1.3 we have $\mathfrak{C l}_{K_{2}}(5) \simeq \operatorname{Gal}\left(H_{2} / K_{2}\right) \simeq M(1) \oplus M\left(\omega^{3}\right)$. In particular $\mathfrak{P}_{1} \sim \mathfrak{P}_{2}^{2} \sim \mathfrak{P}_{3}^{3} \sim \mathfrak{P}_{4}^{4}$. 
We now give a description of the units in $K_{2}$. Substituting $\beta^{5}$ for $\lambda$ in the identity $\left(\lambda-\phi^{5}\right)\left(\lambda-\overline{\phi^{5}}\right)=\lambda^{2}-11 \lambda-1$ we learn $\operatorname{Norm}_{K_{2} \mid \mathbf{Q}}(\beta-\phi)=1$. Thus $\beta-\phi$ is a unit. We choose to work with the units

$$
u_{i}=\frac{\phi \zeta^{i} \beta+1}{\zeta^{i} \beta-\phi} \quad v_{i}=\left(\zeta^{i} \beta\right)^{2}-\zeta^{i} \beta-1 .
$$

It is easy to check

$$
\begin{array}{ll}
\sigma\left(u_{i}\right)=-1 / u_{2 i} & \sigma\left(v_{i}\right)=v_{2 i} \\
\tau\left(u_{i}\right)=u_{i+1} & \tau\left(v_{i}\right)=v_{i+1}
\end{array}
$$

and we have relations $\prod u_{i}=-\phi^{15}, \prod v_{i}=-1$. Thus the subgroups of $K_{2}^{*} / K_{2}^{* 5}$ generated by $u_{1}, u_{2}, u_{3}, u_{4}$ and $v_{1}, v_{2}, v_{3}, v_{4}$ are quotients of the $G$ modules $M\left(\omega^{2}, 4\right)$ and $M(1,4)$.

Lemma 7.4 The extension $K_{1} K_{2} / K_{2}$ has Kummer generator $\phi v_{1} v_{2}^{4} v_{3}^{4} v_{4}$.

Proof. By Lemma 7.1 and Proposition 7.2 it is sufficient to show that the stated element is (i) a 5 th power locally at the prime $\mathfrak{L}$ above 5 , and (ii) not a 5 th power locally at the primes $\mathfrak{P}_{i}$ above 11 . A suitable version of Hensel's lemma shows that $a \in \mathfrak{O}_{K_{2}}$ is a 5 th power locally at $\mathfrak{L}$ if and only if $x^{5} \equiv a$ $\left(\bmod \mathfrak{L}^{26}\right)$ is soluble. We prove $(\mathrm{i})$ by using pari to perform calculations in the group $\left(\mathfrak{O}_{K_{2}} / \mathfrak{L}^{26}\right)^{*}$. Since $\phi v_{1} v_{2}^{4} v_{3}^{4} v_{4} \equiv \phi\left(\bmod \mathfrak{P}_{i}\right)$, claim (ii) is clear without computer calculation. This completes the proof of the lemma. Of course more brutal computer calculations are possible, showing

$$
\phi^{2} \pi_{1} \pi_{2}^{4} \pi_{3}^{4} \pi_{4} \equiv\left(\phi v_{1} v_{2}^{4} v_{3}^{4} v_{4}\right)^{-1} \quad\left(\bmod K_{2}^{* 5}\right)
$$

A consequence of Lemma 7.4 is that $K_{2}\left(\sqrt[5]{v_{1} v_{2}^{2} v_{3}^{3} v_{4}^{4}}\right) / K_{2}$ is unramified at all primes. Furthermore this extension is split at the primes above 5 and 11 . By Proposition 7.2 we deduce

$$
v_{1} v_{2}^{2} v_{3}^{3} v_{4}^{4}=w^{5}
$$

for some $w \in K_{2}$. Multiplying $w$ by a 5 th root of unity if necessary

$$
\begin{array}{ll}
\sigma(w) \equiv w^{3} & \bmod \left\langle v_{1}, v_{2}, v_{3}, v_{4}\right\rangle \\
\tau(w) \equiv w v_{0} & \left(\bmod K_{2}^{* 5}\right) .
\end{array}
$$


Proposition 7.5 The units $\zeta, \phi, u_{1}, u_{2}, u_{3}, u_{4}, v_{1}, v_{2}, v_{3}, w$ generate a subgroup of $\mathfrak{O}_{K_{2}}^{*}$ of index prime to 5 .

Proof. By Dirichlet it suffices to check that the elements listed are independent in $K_{2}^{*} / K_{2}^{* 5}$. There is a $G$-module homomorphism

$$
M:=M(\omega) \oplus M\left(\omega^{2}\right) \oplus M\left(\omega^{2}, 4\right) \oplus M\left(\omega^{3}, 4\right) \rightarrow K_{2}^{*} / K_{2}^{* 5}
$$

where the summands correspond to $\zeta, \phi,\left\langle u_{1}, u_{2}, u_{3}, u_{4}\right\rangle$ and $\left\langle v_{1}, v_{2}, v_{3}, w\right\rangle$. We suppose for a contradiction that this map has non-trivial kernel. Then this kernel meets

$$
\begin{aligned}
\operatorname{ker}(\tau-1 \mid M) & =M(\omega) \oplus M\left(\omega^{2}\right) \oplus M(\omega) \oplus M\left(\omega^{2}\right) \\
& =\left\langle\zeta, \phi, u_{1} u_{2}^{2} u_{3}^{3} u_{4}^{4}, v_{1} v_{2}^{4} v_{3}^{4} v_{4}\right\rangle
\end{aligned}
$$

Dividing into $\sigma$-eigenspaces we learn that one of the elements

$$
\zeta^{i}\left(u_{1} u_{2}^{2} u_{3}^{3} u_{4}^{4}\right)^{j}, \quad \phi^{i}\left(v_{1} v_{2}^{4} v_{3}^{4} v_{4}\right)^{j}
$$

is a 5th power. By Lemmas 3.6 and 7.4 these elements are Kummer generators for $J_{1} J_{2} K_{2} / K_{2}$ and for $K_{1} K_{2}(\sqrt[5]{\phi}) / K_{2}$. This gives the required contradiction.

Remark 7.6 According to pari the field $K_{2}$ has class number 25, whereas $\phi, \beta-\phi$ and its conjugates, generate a subgroup of index 5 in $\mathfrak{O}_{K_{2}}^{*} /$ (torsion). Again this is conditional on the Generalised Riemann Hypothesis.

We apply Propositions 2.1 and 2.2 in the case $F=K_{2}$.

Proposition 7.7 The Selmer groups attached to the 5-isogenies (1) are

$$
\begin{array}{ll}
S\left(A_{0} \rightarrow A_{1} / K_{2}\right) \simeq(\mathbf{Z} / 5 \mathbf{Z})^{8} & S\left(A_{2} \rightarrow A_{0} / K_{2}\right) \simeq(\mathbf{Z} / 5 \mathbf{Z})^{15} \\
S\left(A_{1} \rightarrow A_{0} / K_{2}\right) \simeq(\mathbf{Z} / 5 \mathbf{Z})^{2} & S\left(A_{0} \rightarrow A_{2} / K_{2}\right) \simeq \mathbf{Z} / 5 \mathbf{Z} .
\end{array}
$$

Proof. By Propositions 7.2 and 7.5 the space

$$
\left\{\theta \in K_{2}^{*} / K_{2}^{* 5} \mid \operatorname{ord}_{\mathfrak{p}}(\theta) \equiv 0(\bmod 5) \text { for all } \mathfrak{p}\right\}
$$

has basis $\zeta, \phi, u_{1}, u_{2}, u_{3}, u_{4}, v_{1}, v_{2}, v_{3}, w, \pi_{1}, 1-\zeta$. Here $\pi_{1}$ and $1-\zeta$ are contributions from the class group or "virtual units". We choose characters 
$\left(\mathfrak{O}_{K_{2}} / \mathfrak{P}_{i}\right)^{*} \rightarrow \mathbf{Z} / 5 \mathbf{Z}$ and compute these characters on a basis for (25)

\begin{tabular}{r|cccc} 
& $\mathfrak{P}_{1}$ & $\mathfrak{P}_{2}$ & $\mathfrak{P}_{3}$ & $\mathfrak{P}_{4}$ \\
\hline$\zeta$ & 1 & 3 & 2 & 4 \\
$\phi$ & 2 & 3 & 3 & 2 \\
$u_{i}$ & 3 & 2 & 2 & 3 \\
$v_{i}$ & 0 & 0 & 0 & 0 \\
$w$ & 2 & 4 & 1 & 3 \\
$\pi_{1} \pi_{2}^{2} \pi_{3}^{3} \pi_{4}^{4}$ & 3 & 1 & 4 & 2 \\
$2 \phi-1$ & 2 & 2 & 2 & 2
\end{tabular}

By Proposition 2.1 we deduce

$$
S\left(A_{0} \rightarrow A_{1} / K_{2}\right) \simeq\left\langle u_{1}^{\prime}, u_{2}^{\prime}, u_{3}^{\prime}, u_{4}^{\prime}, v_{1}, v_{2}, v_{3}, w^{\prime}\right\rangle \subset K_{2}^{*} / K_{2}^{* 5}
$$

where $u_{i}^{\prime}=u_{i} \phi$ and $w^{\prime}=w \pi_{1} \pi_{2}^{2} \pi_{3}^{3} \pi_{4}^{4}$. Propositions 2.1 and 7.2 give $S\left(A_{0} \rightarrow\right.$ $\left.A_{2} / K_{2}\right) \simeq \mathbf{Z} / 5 \mathbf{Z}$. The remaining statements follow by Proposition 2.2.

Proposition 7.7 furnishes the estimate rank $A\left(K_{2}\right) \leq 8$. We improve on this by computing the Cassels-Tate pairing on $S\left(A_{0} \rightarrow A_{1} / K_{2}\right)$. As a $G$ module we have

$$
S\left(A_{0} \rightarrow A_{1} / K_{2}\right) \simeq M\left(\omega^{2}, 4\right) \oplus M\left(\omega^{3}, 4\right) .
$$

By [Ca1, $\S 7(5)]$ there is a commutative diagram

$$
\begin{aligned}
& \langle\cdot, \cdot\rangle_{K_{2}} \quad: \quad S\left(A_{0} \rightarrow A_{1} / K_{2}\right) \quad \times \quad S\left(A_{0} \rightarrow A_{1} / K_{2}\right) \quad \rightarrow \quad \mathbf{Q} / \mathbf{Z} \\
& \uparrow \text { cores } \quad \downarrow \text { res } \| \\
& \langle\cdot, \cdot\rangle_{K_{1} K_{2}} \quad: \quad S\left(A_{0} \rightarrow A_{1} / K_{1} K_{2}\right) \quad \times \quad S\left(A_{0} \rightarrow A_{1} / K_{1} K_{2}\right) \rightarrow \mathbf{Q} / \mathbf{Z}
\end{aligned}
$$

where res and cores are the maps $K_{2}^{*} / K_{2}^{* 5} \rightleftarrows\left(K_{1} K_{2}\right)^{*} /\left(K_{1} K_{2}\right)^{* 5}$ induced by the natural inclusion and norm map respectively. We compute $\langle c, d\rangle_{K_{2}}$ where

$$
c=\beta^{4}+3 \beta^{3}+4 \beta^{2}+2 \beta+1, \quad d=\beta^{2}-\beta-1 .
$$

A computer search yields a unit $\gamma \in K_{1} K_{2}$ with $\operatorname{Norm}_{K_{1} K_{2} \mid K_{2}}(\gamma)=c^{2}$. An explicit expression for $55 \gamma$ in terms of $u=\varepsilon(\alpha)$ and $\beta$ is

$$
\begin{aligned}
& \left(271858 \beta^{4}-724855 \beta^{3}+1158870 \beta^{2}-663207 \beta-928521\right) u^{4} \\
& \quad+\left(942679 \beta^{4}-1424893 \beta^{3}+1970490 \beta^{2}-2288047 \beta-1777424\right) u^{3} \\
& \quad+\left(2185832 \beta^{4}-3700207 \beta^{3}+8323117 \beta^{2}-6646061 \beta-10380876\right) u^{2} \\
& \quad+\left(-25520 \beta^{4}+52792 \beta^{3}-893918 \beta^{2}+423925 \beta+1617231\right) u \\
& \quad+\left(1351406 \beta^{4}-2180822 \beta^{3}+4850841 \beta^{2}-4066025 \beta-6097410\right)
\end{aligned}
$$


By Lemma 7.1, the primes above 11 are inert in $K_{1} K_{2} / K_{2}$. Any non-trivial character $\mathbf{F}_{11^{5}}^{*} \rightarrow \mathbf{Z} / 5 \mathbf{Z}$ factors via the norm map $\mathbf{F}_{11^{5}}^{*} \rightarrow \mathbf{F}_{11}^{*}$. So by Proposition 2.1, $\gamma$ belongs to $S\left(A_{0} \rightarrow A_{1} / K_{1} K_{2}\right)$. The prime 5 factors as

$$
\begin{aligned}
& (5)=\mathfrak{L}_{0}^{4} \mathfrak{L}_{1}^{4} \mathfrak{L}_{2}^{4} \mathfrak{L}_{3}^{4} \mathfrak{L}_{4}^{4} \quad \text { in } K_{1} \\
& (5)=\mathfrak{L}^{20} \quad \text { in } K_{2} \\
& (5)=\mathbf{L}_{0}^{20} \mathbf{L}_{1}^{20} \mathbf{L}_{2}^{20} \mathbf{L}_{3}^{20} \mathbf{L}_{4}^{20} \quad \text { in } K_{1} K_{2}
\end{aligned}
$$

with $\mathbf{L}_{i} \mid \mathfrak{L}_{i}$. Proposition 4.5 and the above diagram give

$$
\begin{aligned}
\left\langle c^{2}, d\right\rangle_{K_{2}} & =\langle\gamma, d\rangle_{K_{1} K_{2}} \\
& =\sum_{i=0}^{4} \operatorname{Ind}_{\zeta}(\gamma, d)_{\mathbf{L}_{i}}^{i} \\
& =\sum_{i=0}^{4} \operatorname{Ind}_{\zeta}\left(\gamma_{i}, d\right)_{\mathfrak{L}}^{i}
\end{aligned}
$$

where $\gamma_{i} \in K_{2}$ is chosen $\mathbf{L}_{i}$-adically close to $\gamma$. To do this we use (18) and the binomial theorem to choose $\alpha_{i} \in k \mathfrak{L}_{i}$-adically close to $\alpha$. Then we substitute $\varepsilon\left(\alpha_{i}\right)$ for $u=\varepsilon(\alpha)$ in our expression for $\gamma$.

We compute the Hilbert norm residue symbol $(\cdot, \cdot)_{\mathfrak{L}}$ using the product formula and Euler's criterion. Finally a computer calculation shows

$$
\langle c, d\rangle_{K_{2}} \neq 0 \text {. }
$$

This calculation, together with Lemma 5.4, shows that the Cassels-Tate pairing on (26) is non-degenerate. By Proposition 4.1 it follows that $S\left(A_{1} \stackrel{\times 5}{\longrightarrow}\right.$ $\left.A_{1} / K_{2}\right) \simeq \mathbf{Z} / 5 \mathbf{Z}$. Thus $\operatorname{rank} A\left(K_{2}\right)=0$ and $\amalg\left(A_{1} / K_{2}\right)(5)=0$.

From the exact sequences

$$
\begin{gathered}
A_{0}\left(K_{2}\right) \stackrel{\widehat{\psi}}{\longrightarrow} A_{1}\left(K_{2}\right) \longrightarrow S\left(A_{0} \rightarrow A_{1} / K_{2}\right) \longrightarrow \underset{\amalg}{\longrightarrow}\left(A_{0} / K_{2}\right)[\widehat{\psi}] \longrightarrow 0 \\
0 \longrightarrow \amalg\left(A_{0} / K_{2}\right)[\widehat{\psi}] \longrightarrow \amalg\left(A_{0} / K_{2}\right)(5) \stackrel{\widehat{\psi}}{\longrightarrow} \amalg\left(A_{1} / K_{2}\right)(5)
\end{gathered}
$$

we learn $\amalg\left(A_{0} / K_{2}\right)(5) \simeq(\mathbf{Z} / 5 \mathbf{Z})^{8}$. Proposition 4.1 and the exact sequences

$$
\begin{aligned}
& 0 \longrightarrow S\left(A_{0} \rightarrow A_{2} / K_{2}\right) \longrightarrow S\left(A_{0} \stackrel{\times 5}{\rightarrow} A_{0} / K_{2}\right) \longrightarrow S\left(A_{2} \rightarrow A_{0} / K_{2}\right) \\
& 0 \longrightarrow S\left(A_{2} \rightarrow A_{0} / K_{2}\right) \longrightarrow S\left(A_{2} \stackrel{\times 5}{\longrightarrow} A_{2} / K_{2}\right) \longrightarrow S\left(A_{0} \rightarrow A_{2} / K_{2}\right)
\end{aligned}
$$

show that $S\left(A_{2} \stackrel{\times 5}{\rightarrow} A_{2} / K_{2}\right) \simeq(\mathbf{Z} / 5 \mathbf{Z})^{16}$ and that the Cassels-Tate pairing on this Selmer group has rank 6. Since the multiplication by 25 map on $A_{2}$ factors through $A_{1}$ and $\amalg\left(A_{1} / K_{2}\right)(5)=0$, we deduce

$$
\amalg\left(A_{2} / K_{2}\right)(5) \simeq(\mathbf{Z} / 5 \mathbf{Z})^{6} \oplus(\mathbf{Z} / 25 \mathbf{Z})^{8} .
$$

This completes the proof of Theorem 2. 


\section{References}

[BBBCO] C. Batut, K. Belabas, D. Bernardi, H. Cohen and M. Olivier, pari/gp, a computer algebra package, http://www.parigp-home.de

[B] C.D. Beaver, 5-Torsion in the Shafarevich-Tate group of a family of elliptic curves, Journal of Number Theory 82 (2000) 25-46

[Ca1] J.W.S. Cassels, Arithmetic on curves of genus 1, I. On a conjecture of Selmer, J. Reine Angew. Math. 202 (1959) 52-99

[Ca2] J.W.S. Cassels, Arithmetic on curves of genus 1, IV. Proof of the Hauptvermutung, J. Reine Angew. Math. 211 (1962) 95-112

[Ca3] J.W.S. Cassels, Arithmetic on curves of genus 1, VIII. On conjectures of Birch and Swinnerton-Dyer, J. Reine Angew. Math. 217 (1965) 180199

[CF] J.W.S. Cassels and A. Frohlich, editors, Algebraic Number Theory, Academic Press (1967)

[Co] J. Coates, Fragments of $\mathrm{GL}_{2}$ Iwasawa theory of elliptic curves without complex multiplication, in Arithmetic theory of elliptic curves, Cetraro 1997, Lect. Notes in Math. 1716 Springer (1999)

[CH] J. Coates and S. Howson, Euler characteristics and elliptic curves II, J. Math Soc. Japan 53 (2001) 175-235

[CS] J. Coates and R. Sujatha, Galois cohomology of elliptic curves, TIFR Lect. on Math. 88 Narosa (2000)

[Coh] H. Cohen, Advanced topics in computational number theory, GTM 193 Springer (2000)

[Cr] J.E. Cremona, Algorithms for modular elliptic curves (second edition), Cambridge (1997)

[F0] T.A. Fisher, On 5 and 7 descents for elliptic curves, Cambridge PhD Thesis (2000)

[F1] T.A. Fisher, Some examples of 5 and 7 descent for elliptic curves over Q, J. Eur. Math. Soc., published online 15th February 2001 
[F2] T.A. Fisher, Rational points on $X(11)$ over $\mathbf{Q}\left(\mu_{11}\right)$ via Galois equivariance of the Cassels-Tate pairing, preprint

[G] R. Greenberg, Iwasawa theory for elliptic curves, in Arithmetic theory of elliptic curves, Cetraro 1997, Lect. Notes in Math. 1716 Springer (1999)

[LT] S. Lang and H. Trotter, Frobenius distributions in $\mathrm{GL}_{2}$-extensions, Lect. Notes in Math. 504 Springer (1976)

[Ma] B. Mazur, Rational points of abelian varieties with values in towers of number fields, Invent. Math. 18 (1972) 183-266

[Mc] W.G. McCallum, On the Tate-Shafarevich group of the jacobian of a quotient of the Fermat curve, Invent. Math. 93 (1988) 637-666

[Mi] J.S. Milne, Arithmetic duality theorems, Persp. in Math. 1 Academic Press (1986)

[Se1] J.-P. Serre, Abelian l-adic representations and elliptic curves, Benjamin (1968)

[Se2] J.-P. Serre, Propriétés galoisiennes des points d'ordre fini des courbes elliptiques, Invent. Math. 15 (1972) 259-331

[Si] J.H. Silverman, The arithmetic of elliptic curves, GTM 106 Springer (1986)

[V1] J. Vélu, Courbes elliptiques sur Q ayant bonne réduction en dehors de \{11\}, C. R. Acad. Sc. Paris 273 (1971) 73-75

[V2] J. Vélu, Isogénies entre courbes elliptiques, C. R. Acad. Sc. Paris 273 (1971) 238-241

[W] L.C. Washington, Introduction to cyclotomic fields (second edition), GTM 83 Springer (1997) 